\title{
POINTWISE ESTIMATES FOR RELATIVE FUNDAMENTAL SOLUTIONS OF HEAT EQUATIONS IN $\mathbb{R} \times \mathbb{C}$
}

\author{
ANDREW RAICH
}

\begin{abstract}
Let $p: \mathbb{C} \rightarrow \mathbb{R}$ be a subharmonic, nonharmonic polynomial and $\tau \in \mathbb{R}$ a parameter. Define $\bar{Z}_{\tau p}=\frac{\partial}{\partial \bar{z}}+\tau \frac{\partial p}{\partial \bar{z}}=e^{-\tau p} \frac{\partial}{\partial \bar{z}} e^{\tau p}$, a closed, densely defined operator on $L^{2}(\mathbb{C})$. If $\square_{\tau p}=\bar{Z}_{\tau p} \bar{Z}_{\tau p}^{*}$ and $\widetilde{\square}_{\tau p}=\bar{Z}_{\tau p}^{*} \bar{Z}_{\tau p}$, we solve the heat equations $\partial_{s} u+\square_{\tau p} u=0, u(0, z)=f(z)$ and $\partial_{s} \tilde{u}+\widetilde{\square}_{\tau p} \tilde{u}=0$, $\tilde{u}(0, z)=\tilde{f}(z)$. We write the solutions via heat semigroups and show that the solutions can be written as integrals against distributional kernels. We prove that the kernels are $C^{\infty}$ off of the diagonal $\{(s, z, w): s=0$ and $z=w\}$ and find pointwise bounds for the kernels and their derivatives.
\end{abstract}

\section{INTROdUCtion}

The object of this article is to study the relative fundamental solutions of a class of heat equations on $\mathbb{R} \times \mathbb{C}$ that are motivated by and have applications to questions in several complex variables. We solve each heat equation via a heat semigroup and write the solution as a fractional integral operator. We find the regularity of the integral kernel and find pointwise estimates on the kernel and its derivatives. One additional point of interest is that the infintesimal generator of the semigroup is a magnetic Schrödinger operator whose electric potential is nonpositive, yet the large time behavior of the semigroup is well-controlled.

Let $p: \mathbb{C} \rightarrow \mathbb{R}$ be a subharmonic, nonharmonic polynomial and $\tau \in \mathbb{R}$ a parameter. If $z=x_{1}+i x_{2}$ and $\frac{\partial}{\partial \bar{z}}=\frac{1}{2}\left(\frac{\partial}{\partial x_{1}}+i \frac{\partial}{\partial x_{2}}\right)$, define $\bar{Z}_{\tau p}$ to be the operator

$$
\bar{Z}_{\tau p}=\frac{\partial}{\partial \bar{z}}+\tau \frac{\partial p}{\partial \bar{z}}
$$

and let $Z_{\tau p}=-\bar{Z}_{\tau p}^{*}=\frac{\partial}{\partial z}-\tau \frac{\partial p}{\partial z}$ be the negative of the $L^{2}$-adjoint of $\bar{Z}_{\tau p}$. If $\square_{\tau p}=-\bar{Z}_{\tau p} Z_{\tau p}$ and $\widetilde{\square}_{\tau p}=-Z_{\tau p} \bar{Z}_{\tau p}$, then our goal is to understand the heat equations:

$$
\left\{\begin{array}{l}
\frac{\partial u}{\partial s}+\square_{\tau p} u=0 \\
u(0, z)=f(z)
\end{array}\right.
$$

and

$$
\left\{\begin{array}{l}
\frac{\partial \tilde{u}}{\partial s}+\tilde{\square}_{\tau p} \tilde{u}=0 \\
\tilde{u}(0, z)=\tilde{f}(z) .
\end{array}\right.
$$

2000 Mathematics Subject Classification. Primary 32W30, 32W05, 35K15.

Key words and phrases. Gaussian decay, fundamental solution, heat semigroup, Schrödinger operator, polynomial model, domains of finite type, unbounded weakly pseudoconvex domains. 
We write our solutions

$$
u(s, z)=e^{-s \square_{\tau p}}[f](z)=\int_{\mathbb{C}} H_{\tau p}(s, z, w) f(w) d A(w)
$$

and

$$
\tilde{u}(s, z)=e^{-s \tilde{\square}_{\tau p}}[f](z)=\int_{\mathbb{C}} \tilde{H}_{\tau p}(s, z, w) f(w) d A(w)
$$

where $d A$ is Lebesgue meausure on $\mathbb{C}$. After determining the regularity of the kernels $H_{\tau p}(s, z, w)$ and $\tilde{H}_{\tau p}(s, z, w)$, we obtain pointwise estimates on the two functions and their derivatives.

1.1. Background. Since $\bar{Z}_{\tau p}=e^{-\tau p} \frac{\partial}{\partial \bar{z}} e^{\tau p}$, solving $\bar{Z}_{\tau p} u=f$ in $L^{2}(\mathbb{C})$ is equivalent to solving $\bar{\partial} u=f$ in $L^{2}\left(\mathbb{C}, e^{-2 \tau p}\right)$. When $\tau=1$, the $\bar{Z}_{p}$-problem has been solved in $L^{2}(\mathbb{C})$ [Chr91 and on smoothly bounded domains in $\mathbb{C}\left[\right.$ Ber96]. Christ proves that $G_{p}=\square_{p}^{-1}$ is a well-defined, bounded operator on $L^{2}(\mathbb{C})$, so $R_{p}=Z_{p} G_{p}$ is the relative fundamental solution of $\bar{Z}_{p}$. Christ writes $G_{p}$ and $R_{p}$ as integral operators and finds pointwise bounds on the integral kernels. Christ's techniques involve establishing the $L^{2}$-theory of $\bar{Z}_{p}$ and using integration by parts to establish the decay. Christ proves that the kernels of $G_{p}$ and $R_{p}$ decay exponentially with decay term $e^{-\rho(z, w)}$ where $\rho$ is a metric whose size is governed by the size of the derivatives of $p$. Berndtsson also analyzes $G_{p}$ and $R_{p}$ except that he recognizes that $\square_{p}$ is a magnetic Schrödinger operator of the form $2 \square_{p}=\frac{1}{2}(i \nabla-a)^{2}+V$ where $a=\left(-\frac{\partial p}{\partial x_{2}}, \frac{\partial p}{\partial x_{1}}\right)$ and $V=\frac{1}{2} \triangle p$.

The weighted $\bar{\partial}$-problem in $\mathbb{C}$ is not the only problem in which $\square_{\tau p}$ is the crucial object to understand. Mathematicians have analyzed operators on Hartogs domains in $\mathbb{C}^{n}$ by understanding weighted operators on the base space. The original operators are then reconstructed by Fourier series Lig89, FS91, Ber94]. Recently, on a class of Hartogs domains $\Omega \subset \mathbb{C}^{2}$, Fu and Straube FS02, FS03] establish an equivalence between the compactness of the $\bar{\partial}$-Neumann problem and the blowup of the smallest eigenvalue of $\square_{\tau p}$ as $\tau \rightarrow \infty$. Christ and Fu [CF05] build on the work of Fu and Straube to show that the following are equivalent: compactness of the inverse operator of $\bar{\partial}$ Neumann Laplacian, compactness of the inverse operator of Kohn Laplacian $\square_{b}$, and $b \Omega$ satisfiying property $(P)$.

In Rai05b, we present an alternative method to analyzing $\square_{\tau p}$. We study (10) in the case $\tau>0$ (and $\tau=1$ in particular) and show that (3) solves (11). We prove that $\int_{0}^{\infty} e^{-s \square_{\tau p}} d s=\square_{\tau p}^{-1}$ is welldefined and find pointwise estimates of $H_{\tau p}(s, z, w)$ and its derivatives. The techniques include the spectral theorem, results about one-parameter families (OPF) of operators Rai06, the FeynmanKac-Itô formula from mathematical physics, and an energy inequality. Our results allow us to recover estimates of $G_{p}$ and $R_{p}$. In the cases for which $\rho$ has been computed, the decay in [Chr91] and Rai05b agree.

The motivation of Rai05b, Rai06 is not, however, to reprove Christ's results but rather to use the strategy of Nagel and Stein [NS01, NS04, NS] to understand $e^{-s \square_{b}}$ on (the boundary of) decoupled domains. The boundary of a decoupled domain is a manifold $M=\left\{\left(z_{1}, \ldots, z_{n}\right) \in\right.$ $\left.\mathbb{C}^{n}: \operatorname{Im} z_{n}=P\left(z_{1}, \ldots, z_{n-1}\right)\right\}$ where $P\left(z_{1}, \ldots, z_{n-1}\right)=\sum_{j=1}^{n-1} p_{j}\left(z_{j}\right)$ and $p_{j}$ are subharmonic, nonharmonic polynomials. A first step in this direction is the $n=1$ case in $\mathbb{C}^{2}$. In this case, $M$ is called a polynomial model and Nagel and Stein [NS01] prove rapid decay of the heat kernel. The results in this article and Rai05b] provide machinery to improve the decay estimate to exponential decay. This is the topic of a forthcoming paper. 
If $M$ is a polynomial model, then $M \cong \mathbb{C} \times \mathbb{R}$ and the realization of $\bar{\partial}_{b}$ (defined on $M$ ) on $\mathbb{C} \times \mathbb{R}$ is $\bar{L}=\frac{\partial}{\partial \bar{z}}-i \frac{\partial p}{\partial \bar{z}} \frac{\partial p}{\partial t}$. $\bar{L}$ is translation invariant in $t$, and taking a partial Fourier transform in $t$ produces $\bar{Z}_{\tau p}$. Thus, our understanding of $e^{-s \square_{\tau p}}$ for $\tau \in \mathbb{R}$ will yield results about $e^{-s \square_{b}}$ on $M$. When $n>1$, and $M$ is a decoupled domain, the decoupling causes $\square_{\tau P}$ to act diagonally. If $\vartheta_{q}$ is the set of ordered $q$-tuples $\left(j_{1}, \ldots, j_{q}\right)$ where $1 \leq j_{1}<j_{2}<\cdots<j_{q} \leq n$, it turns out that $\square_{\tau P}\left(\sum_{J \in \vartheta_{q}} \varphi_{J} d \bar{z}_{J}\right)=\sum_{J \in \vartheta_{q}} \square_{J}\left(\varphi_{J}\right) d \bar{z}_{J}$, and each $\square_{J}$ has a further decomposition into a sum of $(n-1)$ commuting operators of the form $\square_{\tau p}$ or $\widetilde{\square}_{\tau p}$. Thus, the study of $\square_{\tau P}$ on $(0, q)$ forms reduces to the study of the $\left(\begin{array}{c}n-1 \\ q\end{array}\right)$ operators $\square_{J}$ for $J \in \vartheta_{q}$. Furthermore, the commutation of the elements $\square_{J}$ makes the study of the heat operators $e^{-s \square_{\tau p}}$ and $e^{-s \widetilde{\square}_{\tau p}}$ critical because if $\square_{J}=\square_{1}+\cdots+\square_{n-1}$, then $e^{-s \square}=\prod_{j=1}^{n-1} e^{-s \square_{j}}$.

1.2. Refinement of the Problem and a Discussion of $\widetilde{\square}_{\tau p}$. When $\tau>0$, we Rai05b] write the solution $u(s, z)$ of (11) as

$$
u(s, z)=\int_{\mathbb{C}} H_{\tau p}(s, z, w) f(w) d A(w),
$$

where $H_{\tau p}(s, z, w)$ is a distributional kernel. We show that $H_{\tau p}$ is smooth (on an appropriate region) and establish pointwise estimates for the kernels. We will extend our previous work and analyze the $\tau<0$ case.

$\square_{\tau p}$ has a special relationship with $\tilde{\square}_{\tau p}$.

$$
\begin{aligned}
\square_{\tau p} & =-\frac{\partial^{2}}{\partial z \partial \bar{z}}+\tau \frac{\partial^{2} p}{\partial z \partial \bar{z}}+\tau^{2} \frac{\partial p}{\partial z} \frac{\partial p}{\partial \bar{z}}+\tau\left(\frac{\partial p}{\partial z} \frac{\partial}{\partial \bar{z}}-\frac{\partial p}{\partial \bar{z}} \frac{\partial}{\partial z}\right) \\
& =-\frac{1}{4} \triangle+\frac{1}{4} \tau \triangle p+\frac{\tau^{2}}{4}|\nabla p|^{2}+\frac{i}{2} \tau\left(\frac{\partial p}{\partial x_{1}} \frac{\partial}{\partial x_{2}}-\frac{\partial p}{\partial x_{2}} \frac{\partial}{\partial x_{1}}\right)
\end{aligned}
$$

while

$$
\begin{aligned}
\widetilde{\square}_{\tau p} & =\frac{\partial^{2}}{\partial z \partial \bar{z}}-\tau \frac{\partial^{2} p}{\partial z \partial \bar{z}}+\tau^{2} \frac{\partial p}{\partial z} \frac{\partial p}{\partial \bar{z}}+\tau\left(\frac{\partial p}{\partial z} \frac{\partial}{\partial \bar{z}}-\frac{\partial p}{\partial \bar{z}} \frac{\partial}{\partial z}\right) \\
& =-\frac{1}{4} \triangle-\frac{1}{4} \tau \Delta p+\frac{\tau^{2}}{4}|\nabla p|^{2}+\frac{i}{2} \tau\left(\frac{\partial p}{\partial x_{1}} \frac{\partial}{\partial x_{2}}-\frac{\partial p}{\partial x_{2}} \frac{\partial}{\partial x_{1}}\right) .
\end{aligned}
$$

The immediate consequence of (6) and (8) is that if $\tilde{p}\left(x_{1}, x_{2}\right)=p\left(x_{2}, x_{1}\right)$, then $\square_{(-\tau) p}=\widetilde{\square}_{\tau \tilde{p}}$. Thus, our problem reduces from understand $\square_{\tau p}$ and $\widetilde{\square}_{\tau p}$ for all $\tau$ to understanding $\square_{\tau p}$ and $\widetilde{\square}_{\tau p}$ for $\tau>0$. Thus, the focus of this paper is to understand (2) when $\tau>0$.

$\widetilde{\square}_{\tau p}$ is inherently more difficult to analyze than $\square_{\tau p}$. As a Schrödinger operator, $\widetilde{\square}_{\tau p}$ has a nonpositive, unbounded potential. From the point of view of parabolic operator theory, the $0^{\text {th }}$ order term can be negative and unbounded. In contrast, $\square_{\tau p}$ has a nonnegative potential and a nonnegative $0^{\text {th }}$ order term. A further complication is that for $\tau>0$, null $\left(\widetilde{\square}_{\tau p}\right) \neq\{0\}$ (and in fact may be infinite dimensional, see $[$ Chr91] $)$ while null $\left(\square_{\tau p}\right)=\{0\}$. In fact, since $\widetilde{\square}_{\tau p}$ has nonnegative eigenvalues and is self-adjoint, it follows from the spectral theorem that $\lim _{s \rightarrow \infty} e^{-s \widetilde{\square}_{\tau p}}=S_{\tau p}$ where $S_{\tau p}$ is the Szegö projection, i.e., the projection of $L^{2}(\mathbb{C})$ onto $\operatorname{null}\left(\bar{Z}_{\tau p}\right)$. A consequence of the nonzero limit is that the kernel of $e^{-s \widetilde{\square}_{\tau p}}$ cannot vanish as $s \rightarrow \infty$. Thus, $\int_{0}^{\infty} e^{-s \widetilde{\square}_{\tau p}} d s$ diverges and cannot be the relative fundamental solution of $\widetilde{\square}_{\tau p} . e^{-s \widetilde{\square}_{\tau p}}\left(I-S_{\tau p}\right)$ functions as the natural replacement for $e^{-s \widetilde{\square}_{\tau p}}$ since $\int_{0}^{\infty} e^{-s \widetilde{\square}_{\tau p}}\left(I-S_{\tau p}\right) d s$ does converge and equals the relative 
fundamental solution of $\widetilde{\square}_{\tau p}$. Thus, we wish to understand the pointwise size estimates of the kernel of $e^{-s \widetilde{\square}_{\tau p}}\left(I-S_{\tau p}\right)$ and its derivatives. Specifically, we will write

$$
e^{-s \widetilde{\square}_{\tau p}}\left(I-S_{\tau p}\right)[f](z)=\int_{\mathbb{C}} \tilde{G}_{\tau p}(s, z, w) f(w) d A(w)
$$

and will analyize $\tilde{G}_{\tau p}(s, z, w)$ and its derivatives.

\section{Definitions and Principal Results}

Let $p$ be a subharmonic, nonharmonic polynomial of degree $2 m$. Let $D(z, r)$ be the Euclidean disk centered at $z$ of radius $r$. Set

$$
a_{j k}^{z}=\frac{1}{j ! k !} \frac{\partial^{j+k} p}{\partial z^{j} \partial \bar{z}^{k}}(z) .
$$

Then $p(w)=\sum a_{j k}^{z}(w-z)^{j} \overline{(w-z)}^{k}$. We define following two "size" functions from the CarnotCarathéodory geometry (see [NSW85) on the polynomial model $M_{p}=\left\{\left(z_{1}, z_{2}\right) \in \mathbb{C}^{2}: \operatorname{Im} z_{2}=\right.$ $\left.p\left(z_{1}\right)\right\}$. Since $\bar{Z}_{\tau p}$ is a partial Fourier transform of $\bar{L}$, it not surprising that geometric elements on $M_{p}$ play a role in our analysis. Let

$$
\Lambda(z, \delta)=\sum_{j, k \geq 1}\left|a_{j k}^{z}\right||\delta|^{j+k}
$$

and

$$
\mu_{p}(z, \delta)=\inf _{j, k \geq 1}\left|\frac{\delta}{a_{j k}^{z}}\right|^{\frac{1}{j+k}} .
$$

The functions also arise in the analysis of magnetic Schrödinger operators with electric potentials She96, She99, Kur00. It follows $\mu(z, \delta)$ is an approximate inverse to $\Lambda(z, \delta)$ if $\delta>0$. This means that if $\delta>0$,

$$
\mu(z, \Lambda(z, \delta)) \sim \delta \text { and } \Lambda(z, \mu(z, \delta)) \sim \delta
$$

We use the notation $a \lesssim b$ if $a \leq C b$ where $C$ is a constant that may depend on the dimension 2 and the degree of $p$. We say that $a \sim b$ if $a \lesssim b$ and $b \lesssim a$. For a little intuition for what $\mu_{p}(z, \delta)$ can look like, if $x=\operatorname{Re} z$ and $p(z)=x^{2 m}$, then $\mu_{p}(z, \delta) \sim \frac{\delta^{1 / 2}}{x^{m-1}}+\delta^{\frac{1}{2 m}}$.

We need to establish notation for adjoints. If $T$ is an operator (either bounded or closed and densely defined) on a Hilbert space with inner product $(\cdot, \cdot)$, let $T^{*}$ be the Hilbert space adjoint of $T$. This means that if $f \in \operatorname{Dom} T$ and $g \in \operatorname{Dom} T^{*}$, then $(f, T g)=\left(T^{*} f, g\right)$. If $U$ is an unbounded domain in some Euclidean space and $T$ is an operator acting on $C_{c}^{\infty}(U)$ or $\mathcal{S}(U)=\left\{\varphi \in C^{\infty}(U)\right.$ : $\varphi$ has rapid decay\}, then we denote $T^{\#}$ as the adjoint in the sense of distributions. This means that if $K$ is a distribution or a Schwartz distribution, then $\left\langle T^{\#} K, \varphi\right\rangle=\langle K, T \varphi\rangle$. Note that if $T$ is not $\mathbb{R}$-valued, $T^{*} \neq T^{\#}$.

Since $\square_{\tau p}$ is self-adjoint in $L^{2}(\mathbb{C})$, it will follow that $H_{\tau p}(s, z, w)=\overline{H_{\tau p}(s, w, z)}$. The relevance of this fact for adjoints is that the operators $\bar{Z}_{\tau p, w}^{\#}$ and $\bar{Z}_{\tau p, w}^{\#}$ are the appropriate differential operators to apply to $H_{\tau p}(s, z, w)$ and $\bar{Z}_{\tau p}^{\#} \neq \bar{Z}_{\tau p}$ or $Z_{\tau p}$. Thus, associated to a polynomial $p$ and the parameter $\tau \in \mathbb{R}$ are the weighted differential operators

$$
\bar{W}_{\tau p, w}=\frac{\partial}{\partial \bar{w}}-\tau \frac{\partial p}{\partial \bar{w}}=e^{\tau p} \frac{\partial p}{\partial \bar{w}} e^{-\tau p} \quad W_{\tau p, w}=\frac{\partial}{\partial w}+\tau \frac{\partial p}{\partial w}=e^{-\tau p} \frac{\partial p}{\partial w} e^{\tau p} .
$$


Observe that $\overline{\left(Z_{\tau p}\right)}=\bar{W}_{\tau p}$ and $\overline{\left(\bar{Z}_{\tau p}\right)}=W_{\tau p}$ and

$$
\bar{Z}_{\tau p}^{\#}=-\bar{W}_{\tau p} \quad \text { and } \quad Z_{\tau p}^{\#}=-W_{\tau p}
$$

Analogously to writing $\frac{\partial}{\partial \bar{z}}=\frac{1}{2}\left(\frac{\partial}{\partial x_{1}}+i \frac{\partial}{\partial x_{2}}\right)$, we let $X_{1}$ and $X_{2}$ denote the "real" and "imaginary" parts of $Z_{\tau p}$, that is,

$$
X_{1}=Z_{\tau p}+\bar{Z}_{\tau p}=\frac{\partial}{\partial x_{1}}+i \tau \frac{\partial p}{\partial x_{2}} \quad X_{2}=i\left(Z_{\tau p}-\bar{Z}_{\tau p}\right)=\frac{\partial}{\partial x_{2}}-i \tau \frac{\partial p}{\partial x_{1}} .
$$

Similarly, we let $U_{1}$ and $U_{2}$ denote the "real" and "imaginary" parts of $W_{\tau p}$. Define

$$
U_{1}=W_{\tau p}+\bar{W}_{\tau p}=\frac{\partial}{\partial x_{1}}-i \tau \frac{\partial p}{\partial x_{2}} \quad U_{2}=i\left(W_{\tau p}-\bar{W}_{\tau p}\right)=\frac{\partial}{\partial x_{2}}+i \tau \frac{\partial p}{\partial x_{1}} .
$$

If $\alpha$ is a multiindex, we will also use the notation that $X^{\alpha}$ is a product of $|\alpha|$ operators of the form $X_{1}$ and $X_{2}$ while $U^{\alpha}$ is a similar product, except with $U_{1}$ and $U_{2}$ replacing $X_{1}$ and $X_{2}$.

We are now ready to state the main results.

Theorem 2.1. Let $p$ be a subharmonic, nonharmonic polynomial and $\tau>0$ a parameter. If $n \geq 0$ and $Y^{\alpha}$ is a composition of $|\alpha|$ operators of the form $Y=\bar{Z}_{\tau p, z}, Z_{\tau p, z}, \bar{W}_{\tau p, w}, W_{\tau p, w}$, then there exist positive constants $c, C_{|\alpha|}, C_{n,|\alpha|}$, so that

$$
\left|Y^{\alpha} \tilde{H}_{\tau p}(s, z, w)\right| \leq C_{|\alpha|} e^{-c \frac{|z-w|^{2}}{s}} \max \left\{\frac{e^{-c \frac{s}{\mu_{p}(w, 1 / \tau)^{2}}} e^{-c \frac{s}{\mu_{p}(z, 1 / \tau)^{2}}}}{s^{1+\frac{1}{2}|\alpha|}}, \frac{e^{-c \frac{|z-w|}{\mu_{p}(z, 1 / \tau)}} e^{-c \frac{|z-w|}{\mu_{p}(w, 1 / \tau)}}}{\mu_{p}(w, 1 / \tau)^{2+|\alpha|}}\right\}
$$

Also, in the cases in which the derivatives annihilate the Szegö kernel, i.e., $\frac{\partial^{n}}{\partial s^{n}} Y^{\alpha} S_{\tau p}(z, w)=0$, the estimate simplifies to

$$
\left|\frac{\partial^{n}}{\partial s^{n}} Y^{\alpha} \tilde{H}_{\tau p}(s, z, w)\right| \leq \frac{C_{n,|\alpha|}}{s^{1+n+\frac{1}{2}|\alpha|}} e^{-c \frac{|z-w|^{2}}{s}} e^{-c \frac{s}{\mu_{p}(w, 1 / \tau)^{2}}} e^{-c \frac{s}{\mu_{p}(z, 1 / \tau)^{2}}} .
$$

The corresponding theorem for estimates of $\tilde{G}_{\tau p}(s, z, w)$ is:

Theorem 2.2. Let $p$ be a subharmonic, nonharmonic polynomial and $\tau>0$ a parameter. If $n \geq 1$ and $Y^{\alpha}$ is a composition of $|\alpha|$ operators of the form $Y=\bar{Z}_{\tau p, z}, Z_{\tau p, z}, \bar{W}_{\tau p, w}, W_{\tau p, w}$, then there exist positive constants $c, C_{|\alpha|}, C_{|\alpha|, n}$ so that

$$
\left|Y^{\alpha} \tilde{G}_{\tau p}(s, z, w)\right| \leq C_{|\alpha|} e^{-c \frac{s}{\mu_{p}(w, 1 / \tau)^{2}}} e^{-c \frac{s}{\mu_{p}(z, 1 / \tau)^{2}}} \max \left\{\frac{e^{-c \frac{|z-w|^{2}}{s}}}{s^{1+\frac{1}{2}|\alpha|}}, \frac{e^{-c \frac{|z-w|}{\mu_{p}(z, 1 / \tau)}} e^{-c \frac{|z-w|}{\mu_{p}(w, 1 / \tau)}}}{\mu_{p}(w, 1 / \tau)^{2+|\alpha|}}\right\}
$$

Also, in the cases in which the derivatives annihilate the Szegö kernel, i.e., $\frac{\partial^{n}}{\partial s^{n}} Y^{\alpha} S_{\tau p}(z, w)=0$, then $\frac{\partial^{n}}{\partial s^{n}} Y^{\alpha} \tilde{H}_{\tau p}(s, z, w)=\frac{\partial^{n}}{\partial s^{n}} Y^{\alpha} \tilde{G}_{\tau p}(s, z, w)$, and the estimate simplifies to

$$
\left|\frac{\partial^{n}}{\partial s^{n}} Y^{\alpha} \tilde{H}_{\tau p}(s, z, w)\right| \leq \frac{C_{n,|\alpha|}}{s^{1+n+\frac{1}{2}|\alpha|}} e^{-c \frac{|z-w|^{2}}{s}} e^{-c \frac{s}{\mu_{p}(w, 1 / \tau)^{2}}} e^{-c \frac{s}{\mu_{p}(z, 1 / \tau)^{2}}} .
$$

Using techniques similar to those in [NS01, Rai05b], we have already showed that $\tilde{G}_{\tau p}, \tilde{H}_{\tau p} \in$ $C^{\infty}((0, \infty) \times \mathbb{C} \times \mathbb{C})$ Rai05a]. Our goal is to find pointwise bounds on $\tilde{H}_{\tau p}$ and $\tilde{G}_{\tau p}$ and their derivatives. 
Remark 2.3. The Szegö kernel $S_{\tau p}$ projects $L^{2}(\mathbb{C})$ onto the kernel of $\bar{Z}_{\tau p}$, so $\bar{Z}_{\tau p, z} S_{\tau p}(z, w)=0$. Since $S_{\tau p}$ is self-adjoint in $L^{2}(\mathbb{C}), W_{\tau p, w} S_{\tau p}(z, w)=0$ as well. Moreover, $S_{\tau p}(z, w)$ has no time dependence, so $\frac{\partial}{\partial s} S_{\tau p}(z, w)=0$ which explains the lack of $s$-derivatives in the first estimate of Theorem 2.1 and Theorem 2.2. Also, using the techniques of Rai05b, NS01, the self-adjointness of $e^{-s \widetilde{\square}_{\tau p}}$ and $S_{\tau p}$ implies $\tilde{H}_{\tau p}(s, z, w)=\overline{\tilde{H}_{\tau p}(s, w, z)}$ and $\tilde{G}_{\tau p}(s, z, w)=\overline{\tilde{G}_{\tau p}(s, w, z)}$ (see [Rai05a] for details).

To help us understand Theorem 2.1] and Theorem 2.2 let us recall results from Rai05b].

Theorem 2.4. Let $p$ be a subharmonic, nonharmonic polynomial and $\tau>0$ a parameter. If $n \geq 0$ and $Y^{\alpha}$ is a product of $|\alpha|$ operators $Y=\bar{Z}_{\tau p, z}, Z_{\tau p, z}$ or $\bar{W}_{\tau p, w}, W_{\tau p, w}$, there exist constants $C_{n,|\alpha|}$, $c$ (independent of $\tau$ ) so that

$$
\left|\frac{\partial^{n}}{\partial s^{n}} Y^{\alpha} H_{\tau p}(s, z, w)\right| \leq \frac{C_{n,|\alpha|}}{s^{1+n+\frac{1}{2}|\alpha|}} e^{-c \frac{|z-w|^{2}}{s}} e^{-c \frac{s}{\mu_{p}(z, 1 / \tau)^{2}}} e^{-c \frac{s}{\mu_{p}(w, 1 / \tau)^{2}}} .
$$

By integrating in $s$, we have the corollary

Corollary 2.5. Let $\tau>0$. Let $Y^{\alpha}$ be a product of $|\alpha|$ operators $Y=\bar{Z}_{\tau p, z}, Z_{\tau p, z}$ or $\bar{W}_{\tau p, w}, W_{\tau p, w}$. Then there exist constants $c, C_{|\alpha|}>0$ so that

$$
\left|Y^{\alpha} R_{\tau p}(z, w)\right| \leq C_{|\alpha|} \begin{cases}|z-w|^{-1-|\alpha|} & |z-w| \leq \mu_{p}\left(z, \frac{1}{\tau}\right) \\ \frac{1}{\mu_{p}(z, 1 / \tau)^{1+|\alpha|}} e^{-c \frac{|z-w|}{\mu_{p}(z, 1 / \tau)}} e^{-c \frac{|z-w|}{\mu_{p}(w, 1 / \tau)}} & |z-w| \geq \mu_{p}\left(z, \frac{1}{\tau}\right)\end{cases}
$$

and

$$
\left|Y^{\alpha} S_{\tau p}(z, w)\right| \leq \frac{C_{|\alpha|}}{\mu_{p}(z, 1 / \tau)^{2+|\alpha|}} e^{-c \frac{|z-w|}{\mu_{p}(z, 1 / \tau)}} e^{-c \frac{|z-w|}{\mu_{p}(w, 1 / \tau)}}
$$

$e^{-s \tilde{\square}_{\tau p}}$ should behave well near $s=0$, and it does; the estimates for $\tilde{H}_{\tau p}(s, z, w)$ agree with the estimates for $H_{\tau p}(s, z, w)$ near $0 . e^{-s \widetilde{\square}_{\tau p}}\left(I-S_{\tau p}\right)$ should behave well as $s \rightarrow \infty$, and we see that $\tilde{G}_{\tau p}(s, z, w)$ has the same exponential decay in $s$ that $H_{\tau p}(s, z, w)$ has. On the other hand, $\lim _{s \rightarrow \infty} e^{-s \widetilde{\square}_{\tau p}}=-\lim _{s \rightarrow 0} e^{-s \widetilde{\square}_{\tau p}}\left(I-S_{\tau p}\right)=S_{\tau p}$, and the estimate for $\tilde{H}_{\tau p}(s, z, w)$ as $s \rightarrow \infty$ and the estimate for $\tilde{G}_{\tau p}(s, z, w)$ near $s=0$ agree with the estimate for $S_{\tau p}(z, w)$. Thus, the decay terms in Theorem 2.1] and Theorem 2.2 are reasonable. In the case when the derivative annihilates $S_{\tau p}(z, w)$, we find that the estimates for the derivatives of $\tilde{H}_{\tau p}(s, z, w)$ and $\tilde{G}_{\tau p}(s, z, w)$ agree and behave similarly to derivatives of $H_{\tau p}(s, z, w)$.

\section{Embedding Theorems and Cancellation Conditions}

In Section 4. we will derive estimates of $Y^{\alpha} \tilde{G}_{\tau p}(s, z, w)$ from results about $H_{\tau p}(s, z, w)$. First, however, we need to prove some cancellation conditions for $H_{\tau p}^{s}$ where $H_{\tau p}^{s}[\varphi]=e^{-s \square_{\tau p}}[\varphi]$. In addition to their importance in this paper, the cancellation conditions complement the size estimates of Theorem 2.4 and Corollary 2.5] and we feel the results in this section are interesting in their own right.

We start by strengthening the Sobolev-embedding theorem from Rai05b. Define

$$
\widetilde{\nabla}=\left(X_{1}, X_{2}\right) \text {. }
$$


Note $\widetilde{\nabla}=\nabla+i\left(\tau \frac{\partial p}{\partial x_{2}},-\tau \frac{\partial p}{\partial x_{1}}\right)$. To prove our weighted Sobolev embedding theorem, we adapt ideas from Ada78.

Theorem 3.1. Let $\Delta=\left(a_{1}, b_{1}\right) \times\left(a_{2}, b_{2}\right) \subset \mathbb{R}^{2}$ be a square of sidelength $\delta$. If $\left(x_{1}, x_{2}\right) \in \Delta$ and if $f \in \mathcal{C}^{2}(\Delta)$, then

$$
\left|f\left(x_{1}, x_{2}\right)\right|^{2} \leq 4\left(\frac{1}{\delta^{2}} \int_{\Delta}|f|^{2}+\int_{\Delta}|\widetilde{\nabla} f|^{2}+\delta^{2} \int_{\Delta}\left|X_{2} X_{1} f\right|^{2}\right) .
$$

Proof. Our first goal is to show that for $\zeta \in\left(a_{2}, b_{2}\right)$,

$$
\int_{a_{1}}^{b_{1}}\left|f\left(y_{1}, \zeta\right)\right|^{2} d y_{1} \leq 2\left(\frac{1}{\delta} \int_{\Delta}|f|^{2}+\delta \int_{\Delta}\left|X_{2} f\right|^{2}\right)
$$

For a continuous function $u\left(y_{1}, y_{2}\right)$, by the Mean Value Theorem for Integrals, there exists $\sigma \in$ $\left(a_{2}, b_{2}\right)$ so that

$$
\int_{\Delta}|u|^{2}=\delta \int_{a_{1}}^{b_{1}}\left|u\left(y_{1}, \sigma\right)\right|^{2} d y_{1}
$$

By the Fundamental Theorem of Calculus and Cauchy-Schwarz, for $\zeta \in\left(a_{2}, b_{2}\right)$ (without loss of generality $\zeta \geq \sigma)$

$$
\left|u\left(y_{1}, \zeta\right)\right|^{2} \leq 2\left(\left|u\left(y_{1}, \sigma\right)\right|^{2}+\delta \int_{\sigma}^{\zeta}\left|\frac{\partial u}{\partial x_{2}}\left(y_{1}, t\right)\right|^{2} d t\right) .
$$

We would like to replace the ordinary derivative in (14) with $X_{2}$. To this end, we define a function $A\left(y_{1}, y_{2}\right)$ so that $A\left(y_{1}, y_{2}\right)=-\int_{\sigma}^{y_{2}} \tau \frac{\partial p}{\partial y_{1}}\left(y_{1}, t\right) d t$. Then $\frac{\partial A}{\partial y_{2}}\left(y_{1}, y_{2}\right)=-\tau \frac{\partial p}{\partial y_{1}}\left(y_{1}, y_{2}\right)$, and if we let $u\left(y_{1}, y_{2}\right)=e^{i A\left(y_{1}, y_{2}\right)} f\left(y_{1}, y_{2}\right)$,

$$
\frac{\partial}{\partial y_{2}}\left(e^{i A\left(y_{1}, y_{2}\right)} f\left(y_{1}, y_{2}\right)\right)=e^{i A\left(y_{1}, y_{2}\right)}\left(\frac{\partial f}{\partial y_{2}}-i \tau \frac{\partial p}{\partial y_{1}} f\right)\left(y_{1}, y_{2}\right)=e^{i A\left(y_{1}, y_{2}\right)} X_{2} f\left(y_{1}, y_{2}\right) .
$$

Thus, (14) can be rewritten as

$$
\left|f\left(y_{1}, \zeta\right)\right|^{2} \leq 2\left(\left|f\left(y_{1}, \sigma\right)\right|^{2}+\delta \int_{\sigma}^{\zeta}\left|X_{2} f\left(y_{1}, t\right)\right|^{2} d t\right) \leq 2\left(\left|f\left(y_{1}, \sigma\right)\right|^{2}+\delta \int_{a_{2}}^{b_{2}}\left|X_{2} f\left(y_{1}, t\right)\right|^{2} d t\right) .
$$

Integrating (15) over $\left(a_{1}, b_{1}\right)$ and using (13), we have

$$
\begin{aligned}
\int_{a_{1}}^{b_{1}}\left|f\left(y_{1}, \zeta\right)\right|^{2} d y_{1} & \leq 2\left(\int_{a_{1}}^{b_{1}}\left|f\left(y_{1}, \sigma\right)\right|^{2} d y_{1}+\delta \int_{\Delta}\left|X_{2} f\right|^{2}\right) \\
& \leq 2\left(\frac{1}{\delta} \int_{\Delta}|f|^{2}+\delta \int_{\Delta}\left|X_{2} f\right|^{2}\right)
\end{aligned}
$$

which is (12).

We now finish the proof. Given $u \in \mathcal{C}^{2}(\Delta)$, by the Mean Value Theorem for Integrals, there exists $\varpi \in\left(a_{1}, b_{1}\right)$ so that

$$
\int_{a_{1}}^{b_{1}}\left|u\left(s, x_{2}\right)\right|^{2} d s=\delta\left|u\left(\varpi, x_{2}\right)\right|^{2}
$$


By the Fundamental Theorem of Calculus and Hölder's inequality (without loss of generality $x_{1}>$ $\varpi)$,

$$
\left|u\left(x_{1}, x_{2}\right)\right|^{2} \leq 2\left(\left|u\left(\varpi, x_{2}\right)\right|^{2}+\delta \int_{\varpi}^{x_{1}}\left|\frac{\partial u}{\partial x_{1}}\left(s, x_{2}\right)\right|^{2} d s\right) .
$$

Similarly to above, we let $B\left(x_{1}, x_{2}\right)=\int_{\varpi}^{x_{1}} \tau \frac{\partial p}{\partial x_{2}}\left(s, x_{2}\right) d s$, so $\frac{\partial B}{\partial x_{1}}\left(x_{1}, x_{2}\right)=\tau \frac{\partial p}{\partial x_{2}}\left(x_{1}, x_{2}\right)$. If we set $u\left(x_{1}, x_{2}\right)=e^{i B\left(x_{1}, x_{2}\right)} f\left(x_{1}, x_{2}\right)$, then analogously to earlier,

$$
\frac{\partial u}{\partial x_{1}}\left(x_{1}, x_{2}\right)=e^{i B\left(x_{1}, x_{2}\right)} X_{1} f\left(x_{1}, x_{2}\right) .
$$

Thus, substituting $e^{i B\left(x_{1}, x_{2}\right)} f\left(x_{1}, x_{2}\right)$ into (17) and using (16) and (12), we have

$$
\begin{aligned}
\left|f\left(x_{1}, x_{2}\right)\right|^{2} & \leq 2\left(\left|f\left(\varpi, x_{2}\right)\right|^{2}+\delta \int_{a_{1}}^{b_{1}}\left|X_{1} f\left(s, x_{2}\right)\right|^{2} d s\right) \\
& =2\left(\frac{1}{\delta} \int_{a_{1}}^{b_{1}}\left|f\left(s, x_{2}\right)\right|^{2} d s+\delta \int_{a_{1}}^{b_{1}}\left|X_{1} f\left(s, x_{2}\right)\right|^{2} d s\right) \\
& \leq 4\left(\frac{1}{\delta^{2}} \int_{\Delta}|f|^{2}+\int_{\Delta}\left|X_{2} f\right|^{2}+\int_{\Delta}\left|X_{1} f\right|^{2}+\delta^{2} \int_{\Delta}\left|X_{2} X_{1} f\right|^{2}\right) .
\end{aligned}
$$

From [Rai05b], we can write $X_{j} X_{k}=A_{j k} \square_{\tau p}$ where $A_{j k}$ is a bounded operator in $L^{2}(\mathbb{C})$, and we can estimate $\|X f\|_{L^{2}(\mathbb{C})} \lesssim \frac{1}{\delta}\|f\|_{L^{2}(\mathbb{C})}+\delta\left\|\square_{\tau p} f\right\|_{L^{2}(\mathbb{C})}$. Combining these facts with Proposition 3.1 and Rai05b, we have the corollary:

Corollary 3.2. Let $\tau>0$. There exists $C>0$ so that if $f \in \mathcal{C}^{2}(D(z, \delta))$ and $\operatorname{supp} f \in D(z, \delta)$, then

$$
|f(z)| \leq \frac{C}{\delta}\left(\|f\|_{L^{2}(D(z, \delta))}+\delta^{2}\left\|\square_{\tau p} f\right\|_{L^{2}(D(z, \delta))}\right) .
$$

We need the following Poincaré lemma.

Proposition 3.3. Let $\varphi \in \mathcal{C}_{c}^{1}(\Delta)$ where $\Delta=\left(a_{1}, b_{1}\right) \times\left(a_{2}, b_{2}\right) \subset \mathbb{R}^{2}$ is a square with sidelength $\delta$. Then

where $j=1$ or 2 .

$$
\|\varphi\|_{L^{2}(\Delta)} \leq \sqrt{2} \delta\left\|X_{j} \varphi\right\|_{L^{2}(\mathbb{C})}
$$

Proof. By the Fundamental Theorem of Calculus and Cauchy-Schwarz,

$$
\left|f\left(x_{1}, x_{2}\right)\right|^{2} \leq 2\left(\left|f\left(x_{1}, \zeta\right)\right|^{2}+\delta \int_{a_{2}}^{b_{2}}\left|\frac{\partial f}{\partial x_{2}}\left(x_{1}, t\right)\right|^{2} d t\right) .
$$

Choosing $\zeta$ outside of $\left(a_{2}, b_{2}\right)$ and integrating over $\Delta$ (in $x_{1}$ and $\left.x_{2}\right)$, we see that

$$
\|f\|_{L^{2}(\Delta)}^{2} \leq 2 \delta^{2}\left\|\frac{\partial f}{\partial x_{2}}\right\|_{L^{2}(\Delta)}^{2} .
$$

As in the proof of Theorem 3.1 define a function $A\left(x_{1}, t\right)=-\int_{0}^{t} \tau \frac{\partial p}{\partial x_{1}}\left(x_{1}, s\right) d s$ so that $\frac{\partial A}{\partial t}\left(x_{1}, t\right)=$ $-\tau \frac{\partial p}{\partial x_{1}}\left(x_{1}, t\right)$. If we let $f\left(x_{1}, x_{2}\right)=e^{i A\left(x_{1}, x_{2}\right)} \varphi\left(x_{1}, x_{2}\right)$, then

$$
\left|\frac{\partial f}{\partial x_{2}}\right|=\left|\frac{\partial \varphi}{\partial x_{2}}-i \tau \frac{\partial p}{\partial x_{1}}\right|=\left|X_{2} \varphi\right|,
$$


and plugging in (19) into (18) finishes the proof for $j=2$. The $j=1$ case is analogous and proven by integrating in $x_{1}$ first.

The next lemma proves a cancellation condition for the heat kernel $H_{\tau p}(s, z, w)$.

Lemma 3.4. Let $\tau>0$ and $\delta>0$ and $\varphi \in \mathcal{C}_{c}^{\infty}(D(z, \delta))$. If $Y^{\alpha}$ is a composition of $|\alpha|$ operators of the form $Y=Z_{\tau p}$ and $\bar{Z}_{\tau p}$, then there exists a constant $C_{|\alpha|}$ so that if $|\alpha|=2 k$ is even,

$$
\left|Y^{\alpha} H_{\tau p}^{s}[\varphi](z)\right| \leq C_{|\alpha|} \delta^{-1}\left(\left\|\square_{\tau p}^{k}[\varphi]\right\|_{L^{2}(\mathbb{C})}+\delta^{2}\left\|\square_{\tau p}^{k+1}[\varphi]\right\|_{L^{2}(\mathbb{C})}\right)
$$

and if $|\alpha|=2 k+1$ is odd,

$$
\left|Y^{\alpha} H_{\tau p}^{s}[\varphi](z)\right| \leq C_{|\alpha|} \delta^{-1}\left(\delta\left\|\square_{\tau p}^{k+1} \varphi\right\|_{L^{2}(\mathbb{C})}+\delta^{3}\left\|\square_{\tau p}^{k+2} \varphi\right\|_{L^{2}(\mathbb{C})}\right)
$$

Proof. From Rai05b] and Rai06], if $|\alpha|=2 k$ is even, then $\left\|Y^{\alpha} \varphi\right\|_{L^{2}(\mathbb{C})} \leq C_{\gamma, p}\left\|\square_{\tau p}^{k} \varphi\right\|_{L^{2}(\mathbb{C})}$. By Corollary 3.2, it follows that

$$
\begin{aligned}
\left|Y^{\alpha} H_{\tau p}^{s}[\varphi](z)\right| & \leq \frac{C}{\delta}\left(\left\|Y^{\alpha} H_{\tau p}^{s}[\varphi]\right\|_{L^{2}(\mathbb{C})}+\delta^{2}\left\|\square_{\tau p} Y^{\alpha} H_{\tau p}^{s}[\varphi]\right\|_{L^{2}(\mathbb{C})}\right) \\
& \leq \frac{C}{\delta}\left(\left\|H_{\tau p}^{s} \square_{\tau p}^{k}[\varphi]\right\|_{L^{2}(\mathbb{C})}+\delta^{2}\left\|H_{\tau p}^{s} \square_{\tau p}^{k+1}[\varphi]\right\|_{L^{2}(\mathbb{C})}\right) \\
& \leq \frac{C}{\delta}\left(\left\|\square_{\tau p}^{k}[\varphi]\right\|_{L^{2}(\mathbb{C})}+\delta^{2}\left\|\square_{\tau p}^{k+1}[\varphi]\right\|_{L^{2}(\mathbb{C})}\right) .
\end{aligned}
$$

The last line uses the fact that $e^{-s \square_{\tau p}}$ is a contraction. The $|\alpha|=2 k+1$ case is similar. If $|\beta|=2 n+1$ for some nonnegative integer $n$, then

$$
\begin{aligned}
\left\|X^{\beta} H_{\tau p}^{s}[\varphi]\right\|_{L^{2}(\mathbb{C})} \leq C \sum_{j=1}^{2}\left\|X_{j} H_{\tau p}^{s} \square_{\tau p}^{n}[\varphi]\right\|_{L^{2}(\mathbb{C})} & \leq \frac{C}{\delta}\left(\left\|H_{\tau p}^{s} \square_{\tau p}^{n}[\varphi]\right\|_{L^{2}(\mathbb{C})}+\delta^{2}\left\|H_{\tau p}^{s} \square_{\tau p}^{n+1}[\varphi]\right\|_{L^{2}(\mathbb{C})}\right) \\
& \leq \frac{C}{\delta}\left(\left\|\square_{\tau p}^{n}[\varphi]\right\|_{L^{2}(\mathbb{C})}+\delta^{2}\left\|\square_{\tau p}^{n+1}[\varphi]\right\|_{L^{2}(\mathbb{C})}\right) .
\end{aligned}
$$

Thus,

$$
\begin{aligned}
\left|Y^{\alpha} H_{\tau p}^{s}[\varphi](z)\right| & \leq \frac{C}{\delta}\left(\left\|Y^{\alpha} H_{\tau p}^{s}[\varphi]\right\|_{L^{2}(\mathbb{C})}+\left\|\square_{\tau p} Y^{\alpha} H_{\tau p}^{s}[\varphi]\right\|_{L^{2}(\mathbb{C})}\right) \\
& \leq \frac{C}{\delta}\left(\delta^{-1}\left\|\square_{\tau p}^{k}[\varphi]\right\|_{L^{2}(\mathbb{C})}+\delta\left\|\square_{\tau p}^{k+1}[\varphi]\right\|_{L^{2}(\mathbb{C})}+\delta^{3}\left\|\square_{\tau p}^{k+2}[\varphi]\right\|_{L^{2}(\mathbb{C})}\right) .
\end{aligned}
$$

Using Proposition 3.3, we are done.

Remark 3.5. It is possible to prove a slightly weaker version of Lemma 3.4 using the correspondence between NIS operators and the one-paramater families (OPF) of operators in Rai06. Nagel and Stein [NS01] prove that the heat operators $e^{-s \square_{b}}$ and $e^{-s \square_{b}}(I-S)$ are nonisotropic smoothing (NIS) operators of order 0 with NIS constants independent of $s$. Thus $H_{\tau p}^{s}$ is an OPF operator of order 0 with OPF constants independent of $s$. A result would then follow immediately. The problem, however, is that we lose control over the number of derivatives needed to bound $\left|Y^{\alpha} H_{\tau p}^{s}[\varphi]\right|$. Also, the definition of NIS operator in [NS01] is different than the definition of NIS operator in [NRSW89] on which the correspondence is based. Since the kernels of $e^{-s \square_{b}}$ and $e^{-s \square_{b}}(I-S)$ are smooth, however, it is trivial to show that $e^{-s \square_{b}}$ and $e^{-s \square_{b}}(I-S)$ are NIS operators in the sense of [NRSW89. Regardless, Lemma 3.4 is a direct and more natural proof.

Recall that $G_{\tau p}$ is the solving operator for $\square_{\tau p}$ when $\tau>0$. 
Lemma 3.6. Let $\tau>0$. Let $\delta>0$ and $\varphi \in \mathcal{C}_{c}^{\infty}(D(z, \delta))$. If $Y^{\alpha}$ is a composition of $|\alpha|$ operators of the form $Y=\bar{Z}_{\tau p}$ or $\bar{Z}_{\tau p}$, then there exists a constant $C_{|\alpha|}$ so that if $|\alpha|=2 k>0$ is even or $|\alpha|=0$ and $\delta \geq \mu_{p}\left(z, \frac{1}{\tau}\right)$, then

$$
\left|Y^{\alpha} G_{\tau p}[\varphi](z)\right| \leq C_{|\alpha|} \delta\left(\left\|\square_{\tau p}^{k} \varphi\right\|_{L^{2}(\mathbb{C})}+\delta^{2}\left\|\square_{\tau p}^{k+1} \varphi\right\|_{L^{2}(\mathbb{C})}\right)
$$

and if $|\alpha|=2 k+1>0$ is odd, then

$$
\left|Y^{\alpha} G_{\tau p}[\varphi](z)\right| \leq C_{|\alpha|} \delta\left(\delta\left\|\square_{\tau p}^{k+1} \varphi\right\|_{L^{2}(\mathbb{C})}+\delta^{3}\left\|\square_{\tau p}^{k+2} \varphi\right\|_{L^{2}(\mathbb{C})}\right) .
$$

If $|\alpha|=0$ and $\delta<\mu_{p}\left(z, \frac{1}{\tau}\right)$

$$
\left|G_{\tau p}[\varphi](z)\right| \leq C_{0} \delta\left(\log \left(\frac{2 \mu_{p}\left(z, \frac{1}{\tau}\right)}{\delta}\right)\|\varphi\|_{L^{2}(\mathbb{C})}+\delta^{2}\left\|\square_{\tau p} \varphi\right\|_{L^{2}(\mathbb{C})}\right) .
$$

Proof. Recall that $G_{\tau p}[\varphi](z)=\int_{0}^{\infty} e^{-s \square_{\tau p}}[\varphi](z) d s$. Thus,

$$
\left|Y^{\alpha} G_{\tau p}[\varphi](z)\right| \leq\left|\int_{0}^{\delta^{2}} Y^{\alpha} e^{-s \square_{\tau p}}[\varphi](z) d s\right|+\left|\int_{\delta^{2}}^{\infty} Y^{\alpha} e^{-s \square_{\tau p}}[\varphi](z) d s\right|
$$

We can estimate the first integral in (20) with Lemma 3.4. If $|\alpha|=2 k$, then

$$
\left|\int_{0}^{\delta^{2}} Y^{\alpha} e^{-s \square_{\tau p}}[\varphi](z) d s\right| \leq \delta^{2} \frac{C_{|\alpha|}}{\delta}\left(\left\|\square_{\tau p}^{k} \varphi\right\|_{L^{2}(\mathbb{C})}+\delta^{2}\left\|\square_{\tau p}^{k+1} \varphi\right\|_{L^{2}(\mathbb{C})}\right) .
$$

The first integral in (20) for the $|\alpha|=2 k+1$ case is handled similarly.

For the tail, we first assume $|\alpha| \geq 1$.

$$
\begin{aligned}
& \left|\int_{\delta^{2}}^{\infty} Y^{\alpha} e^{-s \square_{\tau p}}[\varphi](z) d s\right| \leq \int_{\delta^{2}}^{\infty} \int_{\mathbb{C}}\left|Y_{z}^{\alpha} H_{\tau p}(s, z, w) \varphi(w)\right| d A(w) d s \\
& \quad \leq \int_{\mathbb{C}}|\varphi(w)| \int_{\delta^{2}}^{\infty} \frac{1}{s^{1+\frac{1}{2}|\alpha|}} d s \lesssim \delta\|\varphi\|_{L^{2}(\mathbb{C})} \delta^{-|\alpha|}=\delta^{1-|\alpha|}\|\varphi\|_{L^{2}(\mathbb{C})} .
\end{aligned}
$$

A repeated use of Proposition 3.3 shows

$$
\delta^{-|\alpha|}\|\varphi\|_{L^{2}(\mathbb{C})} \leq 2^{|\alpha| / 2}\left\|X_{2}^{|\alpha|} \varphi\right\|_{L^{2}(\mathbb{C})},
$$

and results from Rai05b allow to change $X_{2}^{\alpha}$ to powers of $\square_{\tau p}$ (as done above). If $|\alpha|=0$, then

$$
\left|\int_{\delta^{2}}^{\infty} e^{-s \square_{\tau p}}[\varphi](z) d s\right| \lesssim \int_{\delta^{2}}^{\infty} \int_{\mathbb{C}} \frac{1}{s} e^{-c \frac{s}{\mu_{p}(z, 1 / \tau)^{2}}} d s \lesssim \delta\|\varphi\|_{L^{2}(\mathbb{C})} \int_{\frac{\delta^{2}}{\mu_{p}(z, 1 / \tau)^{2}}}^{\infty} \frac{1}{r} e^{-r} d r
$$

If $\delta \geq \mu_{p}\left(z, \frac{1}{\tau}\right)$, then (21) is bounded by $\delta\|\varphi\|_{L^{2}(\mathbb{C})}$, but if $\delta<\mu_{p}\left(z, \frac{1}{\tau}\right)$, then estimating the final term in (21),

$$
\int_{\frac{\delta^{2}}{\mu_{p}\left(z, \frac{1}{\tau}\right)^{2}}}^{\infty} \frac{1}{r} e^{-r} d r=\int_{\frac{\delta^{2}}{\mu_{p}\left(z, \frac{1}{\tau}\right)^{2}}}^{1} \frac{1}{r} e^{-r} d r+\int_{1}^{\infty} \frac{1}{r} e^{-r} d r \leq 2 \log \left(\frac{2 \mu_{p}(z, 1 / \tau)}{\delta}\right) .
$$




\section{Estimating $\tilde{G}_{\tau p}(s, z, w)$}

The key to estimating $\tilde{G}_{\tau p}(s, z, w)$ and its derivatives lies in the elementary statement that

$$
\square_{\tau p} \bar{Z}_{\tau p}=-\bar{Z}_{\tau p} Z_{\tau p} \bar{Z}_{\tau p}=\bar{Z}_{\tau p} \widetilde{\square}_{\tau p}
$$

From this statement, we have its corollary, that

$$
\square_{\tau p}^{n} \bar{Z}_{\tau p}=\bar{Z}_{\tau p} \widetilde{\square}_{\tau p}^{n}
$$

and

$$
\frac{(-1)^{n} s^{n} \square_{\tau p}^{n}}{n !} \bar{Z}_{\tau p}=\bar{Z}_{\tau p} \frac{(-1)^{n} s^{n} \widetilde{\square}_{\tau p}^{n}}{n !} .
$$

By the spectral theorem, summing in $n$ proves the first statement of

\section{Proposition 4.1.}

$$
e^{-s \square_{\tau p}} \bar{Z}_{\tau p}=\bar{Z}_{\tau p} e^{-s \widetilde{\square}_{\tau p}}
$$

and

$$
e^{-s \widetilde{\square}_{\tau p}} Z_{\tau p}=Z_{\tau p} e^{-s \square_{\tau p}}
$$

Proof. The second statement follows from the equality $\widetilde{\square}_{\tau p} Z_{\tau p}=-Z_{\tau p} \bar{Z}_{\tau p} Z_{\tau p}=Z_{\tau p} \square_{\tau p}$.

Translating Proposition 4.1 to the kernel side, we see that:

Corollary 4.2. Let $\tau>0$. Let $n \geq 0$ be an integer and $Y_{z}^{\alpha}$ be a product of $|\alpha|$ operators of the form $Y=\bar{Z}_{\tau p, z}, Z_{\tau p, z}$ or $\bar{W}_{\tau p, w}, W_{\tau p, w}$. Then

$$
\frac{\partial^{n}}{\partial s^{n}} Y^{\alpha} \bar{Z}_{\tau p, z} \tilde{H}_{\tau p}(s, z, w)=\frac{\partial^{n}}{\partial s^{n}} Y^{\alpha} \bar{Z}_{\tau p, z} \tilde{G}_{\tau p}(s, z, w)=\frac{\partial^{n}}{\partial s^{n}} Y^{\alpha} \bar{W}_{\tau p, w} H_{\tau p}(s, z, w),
$$

and

$$
\frac{\partial^{n}}{\partial s^{n}} Y^{\alpha} W_{\tau p, w} \tilde{H}_{\tau p}(s, z, w)=\frac{\partial^{n}}{\partial s^{n}} Y^{\alpha} W_{\tau p, w} \tilde{G}_{\tau p}(s, z, w)=\frac{\partial^{n}}{\partial s^{n}} Y^{\alpha} Z_{\tau p, z} H_{\tau p}(s, z, w) .
$$

Also, if $Y^{\alpha}=X_{z}^{\beta} U_{w}^{\gamma}$, then

$$
X_{z}^{\beta} U_{w}^{\gamma} \tilde{G}_{\tau p}(s, z, w)=-\int_{\mathbb{C}} U_{w}^{\gamma} \bar{W}_{\tau p, w} H_{\tau p}(s, v, w) X_{z}^{\beta} R_{\tau p}(z, v) d A(v) .
$$

Proof. The first two equalities follow immediately from interpreting Proposition 4.1] on the kernel side. The third equality requires a short argument. Since

$$
\bar{Z}_{\tau p} e^{-s \widetilde{\square}_{\tau p}}=e^{-s \square_{\tau p}} \bar{Z}_{\tau p},
$$

we can apply $R_{\tau p}$ to both sides and get

$$
\left(I-S_{\tau p}\right) e^{-s \widetilde{\square}_{\tau p}}=R_{\tau p} e^{-s \square_{\tau p}} \bar{Z}_{\tau p} .
$$

The justification that $R_{\tau p} \bar{Z}_{\tau p}=I-S_{\tau p}$ is Chr91, pp.219-220. But $\left(I-S_{\tau p}\right) e^{-s \widetilde{\square}_{\tau p}}=\tilde{G}_{\tau p}^{s}$, and

$$
\begin{aligned}
R_{\tau p} e^{-s \square_{\tau p}\left[\bar{Z}_{\tau p} \varphi\right](z)} & =-R_{\tau p}\left[\int_{\mathbb{C}} \bar{W}_{\tau p, w} H_{\tau p}(s, \cdot, w) \varphi(w) d A(w)\right](z) \\
& =-\int_{C}\left(\int_{\mathbb{C}} R_{\tau p}(z, v) \bar{W}_{\tau p, w} H_{\tau p}(s, v, w) d A(v)\right) \varphi(w) d A(w) .
\end{aligned}
$$


Thus

$$
\tilde{G}_{\tau p}(s, z, w)=-\int_{\mathbb{C}} \bar{W}_{\tau p, w} H_{\tau p}(s, v, w) R_{\tau p}(z, v) d A(v) .
$$

Applying derivatives finishes the proof.

Corollary 4.2 and Theorem 2.4 give immediate estimates for special derivatives of $\tilde{G}_{\tau p}(s, z, w)$ and $\tilde{H}_{\tau p}(s, z, w)$.

Theorem 4.3. Let $p$ be a subharmonic, nonharmonic polynomial and $\tau>0$ a parameter. Let $n \geq 0$ be an integer and $Y^{\alpha}$ a product of $|\alpha|$ operators of the form $Y=\bar{Z}_{\tau p, z}, Z_{\tau p, z}$, or $\bar{W}_{\tau p, w}, W_{\tau p, w}$. If $\frac{\partial^{n}}{\partial s^{n}} Y^{\alpha}$ annihilates the Szegö kernel, i.e., $\frac{\partial^{n}}{\partial s^{n}} Y^{\alpha} S_{\tau p}(z, w)=0$, then there exist constants $c, C_{n,|\alpha|}>0$ (depending on $n$ and $|\alpha|$ ) so that

$$
\left|\frac{\partial^{n}}{\partial s^{n}} Y^{\alpha} \tilde{H}_{\tau p}(s, z, w)\right|=\left|\frac{\partial^{n}}{\partial s^{n}} Y^{\alpha} \tilde{G}_{\tau p}(s, z, w)\right| \leq \frac{C_{|\alpha|}}{s^{1+n+\frac{1}{2}|\alpha|}} e^{-c \frac{|z-w|^{2}}{s}} e^{-c \frac{s}{\mu_{p}(w, 1 / \tau)^{2}}} e^{-c \frac{s}{\mu_{p}(z, 1 / \tau)^{2}}} .
$$

The last result we need to prove Theorem [2.2 is from Chr91 relating the size of $\mu_{p}\left(z, \frac{1}{\tau}\right)$ and $\mu_{p}\left(w, \frac{1}{\tau}\right)$.

Lemma 4.4. If $\tau>0$ and $|z-w|>\mu_{p}\left(w, \frac{1}{\tau}\right)$, then

$$
\frac{\mu_{p}(z, 1 / \tau)}{\mu_{p}(w, 1 / \tau)}+\frac{\mu_{p}(w, 1 / \tau)}{\mu_{p}(z, 1 / \tau)} \leq C\left(\frac{|z-w|}{\mu_{p}(w, 1 / \tau)}\right)^{\operatorname{deg} p}
$$

for some constant $C<\infty$.

One effect of Lemma 4.4 is that in a situation where $|z-w| \geq \mu_{p}\left(z, \frac{1}{\tau}\right)$ (which implies that $\left.|z-w| \gtrsim \mu_{p}\left(w, \frac{1}{\tau}\right)\right)$ we can perform the following estimate

$$
\frac{1}{\mu_{p}\left(z, \frac{1}{\tau}\right)} e^{-c \frac{|z-w|}{\mu_{p}(z, 1 / \tau)}}=\frac{1}{\mu_{p}\left(w, \frac{1}{\tau}\right)} \frac{\mu_{p}(w, 1 / \tau)}{\mu_{p}(z, 1 / \tau)} e^{-c \frac{|z-w|}{\mu_{p}(z, 1 / \tau)}} \lesssim \frac{1}{\mu_{p}\left(w, \frac{1}{\tau}\right)} e^{-c \frac{|z-w|}{\mu_{p}(z, 1 / \tau)}}
$$

with a possible decrease of $c$. (22) reiterates that the statements of Theorem 2.1 and Theorem 2.2 are symmetric in $z$ and $w$.

Proof (Theorem [2.2). We estimate $X_{z}^{\alpha} U_{w}^{\beta} \tilde{G}_{\tau p}(s, z, w)$. This estimate and Theorem 4.3 prove Theorem 2.2. We are now ready to estimate the integral in Corollary 4.2

It is enough to prove

$$
\left|X_{z}^{\alpha} U_{w}^{\beta} \tilde{G}_{\tau p}(s, z, w)\right| \lesssim e^{-c \frac{s}{\mu_{p}(w, 1 / \tau)^{2}}} \max \left\{\frac{e^{-c \frac{|z-w|^{2}}{s}}}{s^{1+\frac{1}{2}(|\alpha|+|\beta|)}}, \frac{e^{-c \frac{|z-w|}{\mu_{p}(z, 1 / \tau)}}}{\mu_{p}(w, 1 / \tau)^{2}}\right\}
$$

because $\tilde{G}_{\tau p}(s, z, w)$ is conjugate symmetric in $z$ and $w$, and all of the estimates we use in the proof of Theorem 2.2 are symmetric in $z$ and $w$.

Let $U_{w}^{\gamma}=U_{w}^{\beta} \bar{W}_{\tau p, w}$. Then $|\gamma| \geq 1$. For $x \in \mathbb{C}$, let $\varphi_{x} \in \mathcal{C}_{c}^{\infty}(\mathbb{C})$ so that $\varphi_{x} \equiv 1$ on $D\left(x, \mu_{p}\left(x, \frac{1}{\tau}\right)\right)$, $\varphi_{x} \equiv 0$ off $D\left(x, 2 \mu_{p}(x, 1)\right)$, and $\left|D^{n} \varphi_{x}\right| \leq c_{n} \mu_{p}\left(x, \frac{1}{\tau}\right)^{-n}$. For $|z-w|>2 \max \left\{\mu_{p}\left(z, \frac{1}{\tau}\right), \mu_{p}\left(w, \frac{1}{\tau}\right)\right\}$, 
we will estimate

$$
\begin{aligned}
\int_{\mathbb{C}} U_{w}^{\beta} \bar{W}_{\tau p, w} H_{\tau p}(s, v, w) & X_{z}^{\alpha} R_{\tau p}(z, v) d A(v)=\underbrace{\int_{\mathbb{C}} U_{w}^{\gamma} H_{\tau p}(s, v, w) X_{z}^{\alpha} R_{\tau p}(z, v) \varphi_{z}(v)\left(1-\varphi_{w}(v)\right) d A(v)}_{I} \\
& +\underbrace{\int_{\mathbb{C}} U_{w}^{\gamma} H_{\tau p}(s, v, w) X_{z}^{\alpha} R_{\tau p}(z, v)\left(1-\varphi_{z}(v)\right) \varphi_{w}(v) d A(v)}_{I I} \\
& +\underbrace{\int_{|v-w|>|v-z|} U_{w}^{\gamma} H_{\tau p}(s, v, w) X_{z}^{\alpha} R_{\tau p}(z, v)\left(1-\varphi_{z}(v)\right)\left(1-\varphi_{w}(v)\right) d A(v)}_{I I I} \\
& +\underbrace{\int_{|v-w|<|v-z|} U_{w}^{\gamma} H_{\tau p}(s, v, w) X_{z}^{\alpha} R_{\tau p}(z, v)\left(1-\varphi_{z}(v)\right)\left(1-\varphi_{w}(v)\right) d A(v)}_{I V} .
\end{aligned}
$$

We start by estimating $|I|$. We use Lemma 3.6. Let $H_{\tau p}^{s, w}(v)=H_{\tau p}(s, v, w)$. If $|\alpha|=2 k-1$ is odd and $\delta=\mu_{p}\left(z, \frac{1}{\tau}\right)$, then

$$
\begin{aligned}
|I| & =\left|X_{z}^{\alpha} R_{\tau p}\left[U_{w}^{\gamma} H_{\tau p}^{s, w} \varphi_{z}\right](w)\right| \\
& \leq c_{|\alpha|}\left(\delta\left\|\square_{\tau p}^{k}\left(U_{w}^{\gamma} H_{\tau p}(s, v, w) \varphi_{z}(v)\right)\right\|_{L^{2}(\mathbb{C})}+\delta^{3}\left\|\square_{\tau p}^{k+1}\left(U_{w}^{\gamma} H_{\tau p}(s, v, w) \varphi_{z}(v)\right)\right\|_{L^{2}(\mathbb{C})}\right) .
\end{aligned}
$$

The two terms are estimated similarly. We present an estimate of the first term.

$$
\begin{aligned}
& \delta\left\|\square_{\tau p}^{k}\left(U_{w}^{\gamma} H_{\tau p}(s, v, w) \varphi_{z}(v)\right)\right\|_{L^{2}(\mathbb{C})} \leq \delta \sum_{|I| \leq 2 k}\left\|X_{v}^{I} U_{w}^{\gamma} H_{\tau p}(s, v, w) D^{2 k-|I|} \varphi_{z}(v)\right\|_{L^{2}(\mathbb{C})} \\
& \lesssim \delta \sum_{|I| \leq 2 k} \frac{1}{s^{1+\frac{1}{2}|I|+\frac{1}{2}|\gamma|}} \delta e^{-c \frac{|z-w|^{2}}{s}} e^{-c \frac{s}{\mu_{p}(w, 1 / \tau)^{2}}} \delta^{-2 k+|I|} \delta \\
& \lesssim \frac{1}{s^{1+\frac{1}{2}(|\alpha|+|\beta|)}} e^{-c \frac{|z-w|^{2}}{s}} e^{-c \frac{s}{\mu_{p}(w, 1 / \tau)^{2}}} .
\end{aligned}
$$

The last line follows immediately from the previous line if $s<\mu_{p}\left(w, \frac{1}{\tau}\right)^{2}$. If $s>\mu_{p}\left(w, \frac{1}{\tau}\right)^{2}$, we can use the decay of $e^{-c \frac{s}{\mu_{p}(w, 1 / \tau)^{2}}}$ to achieve the desired bound. Also, the $|\alpha|$ even case uses the same argument as the $|\alpha|$ odd case.

To estimate $I I$, note that on the support of $\varphi_{w},|v-z| \sim|w-z|$ and $\mu_{p}\left(v, \frac{1}{\tau}\right) \sim \mu_{p}\left(w, \frac{1}{\tau}\right)$. Assume first that $|\gamma|=2 k$ is even. If $s<\mu_{p}\left(w, \frac{1}{\tau}\right)^{2}$ and we set $R_{\tau p}^{z}(v)=R_{\tau p}(z, v)$ and $\delta=\mu_{p}\left(w, \frac{1}{\tau}\right)$, then by Lemma 3.4.

$$
\begin{aligned}
|I I| & =\left|U_{w}^{\gamma}\left(H_{\tau p}^{s}\right)^{\#}\left[X_{z}^{\alpha} R_{\tau p}^{z} \varphi\right](w)\right| \\
& \leq \frac{C}{\delta}\left(\left\|\left(\square_{\tau p, v}^{\#}\right)^{k}\left(X_{z}^{\alpha} R_{\tau p}^{z} \varphi_{w}\right)\right\|_{L^{2}(\mathbb{C})}+\delta^{2}\left\|\left(\square_{\tau p, v}^{\#}\right)^{k+1}\left(X_{z}^{\alpha} R_{\tau p}^{z} \varphi_{w}\right)\right\|_{L^{2}(\mathbb{C})}\right) .
\end{aligned}
$$


We estimate the first term.

$$
\begin{aligned}
\frac{1}{\delta}\left\|\left(\square_{\tau p, v}^{\#}\right)^{k}\left(X_{z}^{\alpha} R_{\tau p}^{z} \varphi_{w}\right)\right\|_{L^{2}(\mathbb{C})} & =\frac{1}{\delta} \sum_{|I| \leq 2 k}\left\|\left(U_{v}^{I} X_{z}^{\alpha} R_{\tau p}(z, v)\right) D^{2 k-I} \varphi_{w}(v)\right\|_{L^{2}(\mathbb{C})} \\
& \lesssim \frac{1}{\delta} e^{-c \frac{|z-w|}{\mu_{p}(z, 1 / \tau)}} \frac{1}{\mu_{p}(z, 1 / \tau)^{|\alpha|+1} \mu_{p}\left(w, \frac{1}{\tau}\right)^{|I|}} \mu_{p}(w, 1 / \tau)^{|I|-|\gamma|} \delta \\
& \leq e^{-c \frac{|z-w|}{\mu_{p}(z, 1 / \tau)}} \frac{1}{\mu_{p}(z, 1 / \tau)^{|\alpha|+1} \mu_{p}(w, 1 / \tau)^{|\beta|+1}} .
\end{aligned}
$$

The second term is handled similarly, as is the $|\gamma|$ odd case. If $s \geq \mu_{p}\left(w, \frac{1}{\tau}\right)^{2}$, we would like the estimate of $I I$ as above except with an additional $e^{-c \frac{s}{\mu_{p}(w, 1 / \tau)^{2}}}$ term. For this estimate, we just use the size conditions for $H_{\tau p}$ and $R_{\tau p}$.

$$
|I I| \lesssim e^{-c \frac{|z-w|}{\mu_{p}(z, 1 / \tau)}} \frac{1}{\mu_{p}\left(z, \frac{1}{\tau}\right)^{1+|\alpha|}} \frac{1}{s^{1+\frac{1}{2}|\gamma|}} e^{-c \frac{s}{\mu_{p}(w, 1 / \tau)^{2}}} \mu_{p}\left(w, \frac{1}{\tau}\right)^{2} \leq \frac{e^{-c \frac{|z-w|}{\mu_{p}(z, 1 / \tau)}} e^{-c \frac{s}{\mu_{p}(w, 1 / \tau)^{2}}}}{\mu_{p}(z, 1 / \tau)^{|\alpha|+1} \mu_{p}(w, 1 / \tau)^{|\beta|+1}} .
$$

Estimating $I I I$ and $I V$ follows from the size estimates for $R_{\tau p}$ and $H_{\tau p}$ from Theorem 2.4 and Corollary 2.5. Note that if $|v-w|>|v-z|$, then $|v-w| \geq \frac{1}{2}|z-w|$ and

$$
\begin{aligned}
& |I I I| \leq \int_{\substack{|v-w|>\mu_{p}(w, 1 / \tau) \\
|v-z|>\mu_{p}(z, 1 / \tau) \\
|v-w|>|v-z|}}\left|U_{w}^{\gamma} H_{\tau p}(s, v, w) X_{z}^{\alpha} R_{\tau p}(z, v)\right| d A(v) \\
& \lesssim \frac{1}{s^{1+\frac{1}{2}|\gamma|}} e^{-c \frac{|z-w|^{2}}{s}} e^{-c \frac{s}{\mu_{p}(w, 1 / \tau)^{2}}} \int_{|v-z|>\mu_{p}(z, 1 / \tau)} \frac{1}{\mu_{p}\left(z, \frac{1}{\tau}\right)^{|\alpha|+1}} e^{-c \frac{|z-v|}{\mu_{p}(z, 1 / \tau)}} d A(v) \\
& \leq \frac{1}{s^{1+\frac{1}{2}|\beta|+\frac{1}{2}}} \frac{1}{\mu_{p}(z, 1 / \tau)^{|\alpha|-1}} e^{-c \frac{|z-w|^{2}}{s}} e^{-c \frac{s}{\mu_{p}(w, 1 / \tau)^{2}}} .
\end{aligned}
$$

(23) is close the desired estimated, but we need to replace $\frac{1}{\mu_{p}(z, 1 / \tau)^{|\alpha|-1}}$ with $\frac{1}{s^{\frac{1}{2}|\alpha|-\frac{1}{2}}}$. There are two cases. If $s>\mu_{p}\left(z, \frac{1}{\tau}\right)^{2}$, then (shrinking $c$ as necessary) by Lemma 4.4 .

$$
\begin{aligned}
& \frac{1}{\mu_{p}\left(z, \frac{1}{\tau}\right)^{|\alpha|-1}} e^{-c \frac{|z-w|^{2}}{s}} e^{-c \frac{s}{\mu_{p}(w, 1 / \tau)^{2}}} \lesssim s^{\frac{1}{2}} \frac{1}{\mu_{p}\left(z, \frac{1}{\tau}\right)^{|\alpha|}} \frac{\mu_{p}\left(w, \frac{1}{\tau}\right)^{|\alpha|}}{s^{\frac{1}{2}|\alpha|}} e^{-c \frac{|z-w|^{2}}{s}} e^{-c \frac{s}{\mu_{p}(w, 1 / \tau)^{2}}} \\
& \lesssim \frac{1}{s^{\frac{1}{2}|\alpha|-\frac{1}{2}}}\left(\frac{|z-w|}{\mu_{p}\left(w, \frac{1}{\tau}\right)}\right)^{M}\left(\frac{s}{|z-w|^{2}}\right)^{\frac{M}{2}}\left(\frac{\mu_{p}\left(w, \frac{1}{\tau}\right)^{2}}{s}\right)^{\frac{M}{2}} e^{-c \frac{|z-w|^{2}}{s}} e^{-c \frac{s}{\mu_{p}(w, 1 / \tau)^{2}}} \\
& \lesssim \frac{1}{s^{\frac{1}{2}|\alpha|-\frac{1}{2}}} e^{-c \frac{|z-w|^{2}}{s}} e^{-c \frac{s}{\mu_{p}(w, 1 / \tau)^{2}}} .
\end{aligned}
$$

If $s<\mu_{p}\left(z, \frac{1}{\tau}\right)$, the estimate is simpler because $\mu_{p}\left(z, \frac{1}{\tau}\right)<|w-z|$, and we can use the Gaussian decay term to achieve the desired bound. 
To estimate $I V$, note that if $|v-w|<|v-z|$, then $|v-z| \geq \frac{1}{2}|w-z|$, so

$$
\begin{aligned}
|I V| & \leq \int_{\substack{|v-w|>\mu_{p}(w, 1 / \tau) \\
|v-z|>\mu_{p}(z, 1 / \tau) \\
|v-w|<|v-z|}}\left|U_{w}^{\gamma} H_{\tau p}(s, v, w) X_{z}^{\alpha} R_{\tau p}(z, v)\right| d A(v) \\
& \lesssim \frac{1}{s^{1+\frac{1}{2}|\gamma|}} e^{-c \frac{s}{\mu_{p}(w, 1 / \tau)^{2}}} \frac{e^{-c \frac{|z-w|}{\mu_{p}(z, 1 / \tau)}}}{\mu_{p}\left(z, \frac{1}{\tau}\right)^{1+|\alpha|}} \int_{|v-w|>\mu_{p}\left(w, \frac{1}{\tau}\right)} e^{-c \frac{|v-w|^{2}}{s}} d A(v) \\
& \lesssim \frac{1}{s^{1+\frac{1}{2}|\gamma|}} e^{-c \frac{s}{\mu_{p}(w, 1 / \tau)^{2}}} \frac{e^{-c \frac{|z-w|}{\mu_{p}(z, 1 / \tau)}}}{\mu_{p}\left(z, \frac{1}{\tau}\right)^{1+|\alpha|}} \int_{\mu_{p}(w, 1 / \tau)}^{\infty} s e^{-c \frac{r^{2}}{s}} \frac{r}{s} d r \\
& \sim \frac{1}{s^{\frac{1}{2}|\gamma|}} e^{-c \frac{s}{\mu_{p}(w, 1 / \tau)^{2}}} \frac{e^{-c \frac{|z-w|}{\mu_{p}(z, 1 / \tau)}}}{\mu_{p}\left(z, \frac{1}{\tau}\right)^{1+|\alpha|}} e^{-c \frac{\mu_{p}(w, 1 / \tau)^{2}}{s}}
\end{aligned}
$$

(24) easily reduces to the desired estimate.

We now have to worry about the $|z-w| \leq 2 \mu_{p}\left(z, \frac{1}{\tau}\right)$ case. Note that if $|z-w| \leq 2 \mu_{p}\left(z, \frac{1}{\tau}\right)$, then $\mu_{p}\left(w, \frac{1}{\tau}\right) \sim \mu_{p}\left(z, \frac{1}{\tau}\right)$. Similarly to above, let $\psi_{z} \in \mathcal{C}_{c}^{\infty}(\mathbb{C})$ with $\operatorname{supp} \psi \subset D\left(z, 4 \mu_{p}(z, 1 / \tau)\right), \psi \equiv 1$ on $D\left(z, 2 \mu_{p}(z, 1 / \tau)\right)$, and $\left|D^{k} \psi\right| \leq c_{k} \mu_{p}(z, 1 / \tau)^{-k}$. Then

$$
\begin{aligned}
& \int_{\mathbb{C}} U_{w}^{\gamma} H_{\tau p}(s, v, w) X_{z}^{\alpha} R_{\tau p}(z, v) d A(v) \\
& =\int_{\mathbb{C}} U_{w}^{\gamma} H_{\tau p}(s, v, w) X_{z}^{\alpha} R_{\tau p}(z, v) \psi_{z}(v) d A(v)+\int_{\mathbb{C}} U_{w}^{\gamma} H_{\tau p}(s, v, w) X_{z}^{\alpha} R_{\tau p}(z, v)\left(1-\psi_{z}(v)\right) d A(v)
\end{aligned}
$$

The first term in the above equality can be estimated using the cancellation condition for $R_{\tau p}$, Lemma 3.6. This estimate is the same as the estimate of $I$. To bound the second term, we can split the integral up with regions $|v-w|>|v-z|$ and $|v-w|<|v-z|$. In this manner, the estimate is very similar to the estimates for $I I I$ and $I V$.

\section{Off-diagonal estimates of $\tilde{H}_{\tau p}(s, z, w)$}

We prove an off-diagonal Gaussian decay estimate for $\tilde{H}_{\tau p}(s, z, w)$. On-diagonal Gaussian estimates, i.e., estimates where $|z-w| \leq \sqrt{s}$, are false since $\lim _{s \rightarrow \infty} \tilde{H}_{\tau p}(s, z, w)=S_{\tau p}(z, w)$. The $1 / s$ term in Gaussian decay would force $\lim _{s \rightarrow \infty} \tilde{H}_{\tau p}(s, z, w)=0$ which clearly does not occur.

We need some notation. Let $E^{\widetilde{\square}_{\tau p}}$ be the resolution of the identity for $\widetilde{\square}_{\tau p}$ (see [Rud91] or [RS80] for details). By the spectral theorem, the operator

$$
F\left(\widetilde{\square}_{\tau p}\right)=\int_{0}^{\infty} F(\lambda) d E^{\widetilde{\square}_{\tau p}}(\lambda) .
$$

is defined so that given $f_{1}, f_{2} \in L^{2}(\mathbb{C}), d E_{f_{1}, f_{2}}^{\widetilde{\square}_{\tau p}}$ is a measure with support on $\sigma\left(\widetilde{\square}_{\tau p}\right) \subset[0, \infty)$ and

$$
\left(F\left(\widetilde{\square}_{\tau p}\right) f_{1}, f_{2}\right)=\int_{0}^{\infty} F(\lambda) d E_{f_{1}, f_{2}}^{\widetilde{\square}_{\tau p}} .
$$

Our integral operators $F\left(\widetilde{\square}_{\tau p}\right)$ can be given by integration against a kernel, and we denote the kernel of $F\left(\widetilde{\square}_{\tau p}\right)$ by $K_{F}(z, w)$. For example, $K_{e^{-s \tilde{\square}_{\tau p}}}(z, w)=\tilde{H}_{\tau p}(s, z, w)$. 
The idea of the argument is to decompose $e^{-s \widetilde{\square}_{\tau p}}=F_{\ell}^{0}\left(\sqrt{s \widetilde{\square}_{\tau p}}\right)+F_{\ell}^{\infty}\left(\sqrt{s \widetilde{\square}_{\tau p}}\right)$. We will fix $(s, z, w)$ and choose $\ell$ and $F_{\ell}^{0}$ appropriately so that $K_{F_{\ell}^{0}}(z, w)=0$. The decomposition is based on a finite propogation speed result for operators which can be written in terms of the wave kernel $\cos \left(\sqrt{s \widetilde{\square}_{\tau p}}\right)$. The "cut-off" introduces a Gaussian decay term into $F_{\ell}^{\infty}$, and a good poinwise estimate of the tail of the Fourier transform of a Gaussian allows us to recover the off-diagonal Gaussian decay estimate for $\tilde{H}_{\tau p}(s, z, w)$. In addition to the tail estimate, we also need $L^{2}$-estimates on the integral kernel of the resolvant $R\left(\ell, \widetilde{\square}_{\tau p}\right)=\left(\ell I-\widetilde{\square}_{\tau p}\right)^{-1}$, and $L^{2}$-estimates on the kernel of a composition of operators.

5.1. Finite Propogation Speed. The finite propogation speed of solutions to the wave equation will follow from a local conservation of energy law.

Proposition 5.1. Fix $\left(s_{0}, z_{0}\right) \in(0, \infty) \times \mathbb{C}$. Let $u$ solve the wave equation

$$
\frac{\partial^{2} u}{\partial s^{2}}+\widetilde{\square}_{\tau p} u=0
$$

in $\left\{(s, z): 0 \leq s \leq s_{0}\right.$ and $\left.z \in \overline{D\left(z_{0}, s_{0}-s\right)}\right\}$. If

$$
e[u](s)=\int_{D\left(z_{0}, s_{0}-s\right)}\left|u_{s}(z)\right|^{2}+\left|\bar{Z}_{\tau p} u(z)\right|^{2} d A(z),
$$

then $e[u](s) \leq e[u](0)$.

Proof. It's enough to show $\dot{e}[u](s) \leq 0$ for $0<s<s_{0}$. If $d S$ is "surface area" measure on the circle with outward unit normal $\nu$, we have:

$$
\begin{aligned}
& \dot{e}[u](s)=\int_{D\left(z_{0}, s_{0}-s\right)} \frac{\partial}{\partial s} u_{s} \bar{u}_{s}+\frac{\partial}{\partial s}\left(\bar{Z}_{\tau p} u \overline{\bar{Z}}_{\tau p} u\right) d A(z)-\int_{\partial D\left(z_{0}, s_{0}-s\right)}\left|u_{s}\right|^{2}+\left|\bar{Z}_{\tau p} u\right|^{2} d S \\
& =2 \int_{D\left(z_{0}, s_{0}-s\right)} \operatorname{Re}\left(u_{s s} \bar{u}_{s}+\bar{Z}_{\tau p} u_{s} \overline{\bar{Z}_{\tau p} u}\right)-\int_{\partial D\left(z_{0}, s_{0}-s\right)}\left|u_{s}\right|^{2}+\left|\bar{Z}_{\tau p} u\right|^{2} d S \\
& =2 \operatorname{Re}\left(\int_{D\left(z_{0}, s_{0}-s\right)} u_{s s} \bar{u}_{s}+\widetilde{\square}_{\tau p} u \bar{u}_{s} d A(z)+\int_{\partial D\left(z_{0}, s_{0}-s\right)} u_{s} \overline{\bar{Z}}_{\tau p} u \nu d S\right)-\int_{\partial D\left(z_{0}, s_{0}-s\right)}\left|u_{s}\right|^{2}+\left|\bar{Z}_{\tau p} u\right|^{2} d S \\
& =2 \operatorname{Re}\left(\int_{D\left(z_{0}, s_{0}-s\right)} \bar{u}_{s}(\underbrace{u_{s s}+\widetilde{\square}_{\tau p} u}_{=0})+\int_{\partial D\left(z_{0}, s_{0}-s\right)} u_{s} \overline{\bar{Z}}_{\tau p} u \nu d S\right)-\int_{\partial D\left(z_{0}, s_{0}-s\right)}\left|u_{s}\right|^{2}+\left|\bar{Z}_{\tau p} u\right|^{2} d S \\
& =-\int_{\partial D\left(z_{0}, s_{0}-s\right)}\left|u_{s}\right|^{2}+\left|\bar{Z}_{\tau p} u\right|^{2} d S+2 \int_{\partial D\left(z_{0}, s_{0}-s\right)} \operatorname{Re}\left(u_{s} \overline{\bar{Z}_{\tau p} u}\right) \nu d S \\
& \leq-\int_{\partial D\left(z_{0}, s_{0}-s\right)}\left|u_{s}\right|^{2}+\left|\bar{Z}_{\tau p} u\right|^{2} d S+2 \int_{\partial D\left(z_{0}, s_{0}-s\right)}\left|u_{s} \overline{\bar{Z}_{\tau p} u}\right| d S \\
& \leq-\int_{\partial D\left(z_{0}, s_{0}-s\right)}\left|u_{s}\right|^{2}+\left|\bar{Z}_{\tau p} u\right|^{2} d S+2 \int_{\partial D\left(z_{0}, s_{0}-s\right)} \frac{1}{2}\left|u_{s}\right|^{2}+\frac{1}{2}\left|\bar{Z}_{\tau p} u\right|^{2} d S \leq 0 .
\end{aligned}
$$

We have the following corollary. 
Corollary 5.2. Fix $\left(s_{0}, z_{0}\right) \in(0, \infty) \times \mathbb{C}$. Let $u$ solve the wave equation

$$
\frac{\partial^{2} u}{\partial s^{2}}+\widetilde{\square}_{\tau p} u=0
$$

in $\left\{(s, z): 0 \leq s \leq s_{0}\right.$ and $\left.z \in \overline{D\left(z_{0}, s_{0}-s\right)}\right\}$. If $u(0, z)=\frac{\partial u}{\partial s}(0, z)=0$ on $D\left(z_{0}, s_{0}\right)$, then

$$
u(s, z) \equiv 0
$$

on the cone

$$
\left\{(s, z): 0 \leq s \leq s_{0}:\left|z-z_{0}\right|<s_{0}-s\right\}
$$

Proof. From Lemma 5.1

$$
\int_{D\left(z_{0}, s_{0}-s\right)}\left|u_{s}(s, z)\right|^{2}+\left|\bar{Z}_{\tau p} u(s, z)\right|^{2} d A(z) \leq \int_{D\left(z_{0}, s_{0}\right)}\left|u_{s}(0, z)\right|^{2}+\left|\bar{Z}_{\tau p} u(0, z)\right|^{2} d A(z) .
$$

However, $u_{s}(0, z)=0$ on $D\left(z_{0}, s_{0}\right)$. Also, since $u(0, z)=0$ in $D\left(z_{0}, s_{0}\right), \bar{Z}_{\tau p} u(0, z)=0$ in $D\left(z_{0}, s_{0}-\right.$ $s)$. The corollary follows quickly.

Let $f$ be a suitably nice function defined on $\mathbb{C}$. Use the spectral theorem to define $V_{\tau p} f(s, z)=$ $\cos \left(s \sqrt{\widetilde{\square}_{\tau p}}\right)[f](z) . V_{\tau p}$ is a contraction on $L^{2}(\mathbb{C})$, so we can write

$$
V_{\tau p}[f](s, z)=\int_{\mathbb{C}} V_{\tau p}(s, z, w) f(w) d A(w)
$$

for some distributional kernel $V_{\tau p}(s, z, w)$. In our new notation, Corollary 5.2 becomes

Corollary 5.3. If $f(z)=0$ on $D\left(z_{0}, s_{0}\right)$, then

$$
V_{\tau p} f(s, z) \equiv 0
$$

on the cone

$$
\left\{(s, z): 0 \leq s \leq s_{0}:\left|z-z_{0}\right|<s_{0}-s\right\}
$$

The finite propagation speed of solutions to the wave equation implies a certain support of the wave kernel $V_{\tau p}(s, z, w)$.

\section{Corollary 5.4.}

$$
V_{\tau p}(s, z, w)=0 \text { if }|z-w|>s .
$$

Proof. Fix $s \in[0, \infty)$ and $z \in \mathbb{C}$. Let $f \in C_{c}^{\infty}(\mathbb{C})$ be defined so that $\operatorname{supp} f \subset \mathbb{C} \backslash D(z, s)$. Then with $s_{0}=s$ and $z_{0}=z$, Corollary 5.3 shows $V_{\tau p} f(s, z)=0$. As a result

$$
\begin{aligned}
0 & =V_{\tau p} f(s, z)=\int_{D(z, s)} V_{\tau p}(s, z, w) f(w) d A(w)+\int_{\mathbb{C} \backslash D(z, s)} V_{\tau p}(s, z, w) f(w) d A(w) \\
& =\int_{\mathbb{C} \backslash D(z, s)} V_{\tau p}(s, z, w) f(w) d A(w) .
\end{aligned}
$$

Since $f$ was arbitrary, $V_{\tau p}(s, z, w)=0$ on $\{w \in \mathbb{C} \backslash D(z, s)\}$.

The finite propogation speed of the wave kernel allows us to adapt Sikora's technique Sik04 for off-diagonal Gaussian estimates. 
5.2. Spectral Theorem Facts. Now we collect some useful results. The support condition of the wave kernel has the following consequence which is a crucial tool for our decomposition of $e^{-s \widetilde{\square}_{\tau p}}$.

Proposition 5.5. Let $F$ be a bounded, even function defined on $\mathbb{R}$ whose Fourier transform satisfies $\operatorname{supp} \hat{F} \subset[-L, L]$. Then $K_{F\left(\sqrt{s \widetilde{\square}_{\tau p}}\right)}(z, w)=0$ if $|z-w|>s^{1 / 2} L$.

Proof. Since $F$ is even, the Fourier inversion formula can be written $F(\sqrt{x})=\frac{1}{2 \pi} \int_{\mathbb{R}} \hat{F}(t) \cos (t \sqrt{x}) d t$, and the spectral theorem implies

$$
F\left(\sqrt{s \widetilde{\square}_{\tau p}}\right)=\frac{1}{2 \pi} \int_{\mathbb{R}} \hat{F}(t) \cos \left(t \sqrt{s \widetilde{\square}_{\tau p}}\right) d t .
$$

Thus, for $f_{1}, f_{2} \in C_{c}^{\infty}(\mathbb{C})$,

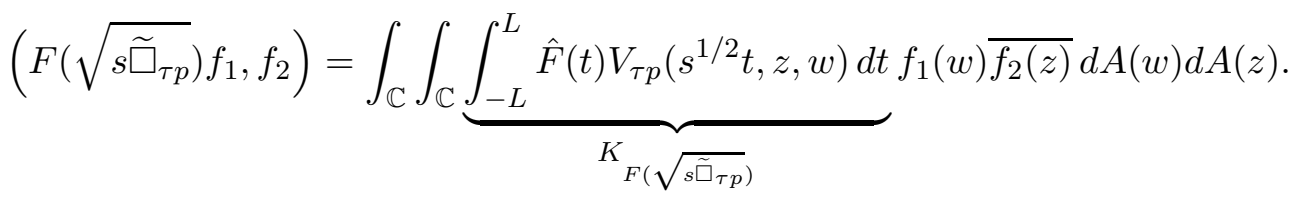

Proposition 5.5 follows quickly from (25) and Corollary 5.4

Next we investigate products.

Proposition 5.6. If $F$ and $G$ are bounded functions on $\mathbb{R}$,

$$
\left\|K_{F G}(z, \cdot)\right\|_{L^{2}(\mathbb{C})} \leq\|G\|_{L^{\infty}(\mathbb{C})}\left\|K_{F}(z, \cdot)\right\|_{L^{2}(\mathbb{C})} .
$$

Proof. Since $G$ is bounded, $G\left(\widetilde{\square}_{\tau p}\right)=\int_{0}^{\infty} G(\lambda) d E^{\widetilde{\square}_{\tau p}}(\lambda)$, and it is clear that $\left\|G\left(\widetilde{\square}_{\tau p}\right)\right\|_{L^{2} \rightarrow L^{2}} \leq$ $\|G\|_{L^{\infty}(\mathbb{C})}$. Also, fixing $z, w \in \mathbb{C}$ and writing $K_{F}(z, v)=K_{F}^{z}(v)$ to emphasize that $z$ is fixed, we have

$$
K_{F G}(z, w)=\int_{\mathbb{C}} K_{F}(z, v) K_{G}(v, w) d A(v)=\int_{\mathbb{C}} K_{F}^{z}(v) K_{G}(v, w) d A(v)=G^{*}\left(\sqrt{\widetilde{\square}_{\tau p}}\right)\left[K_{F}^{z}\right](w) .
$$

But $\|G\|_{L^{2} \rightarrow L^{2}}=\left\|G^{*}\right\|_{L^{2} \rightarrow L^{2}}$, so

$$
\left\|K_{F G}(z, \cdot)\right\|_{L^{2}(\mathbb{C})}=\left\|G^{*}(\sqrt{\square})\left[K_{F}^{z}\right]\right\|_{L^{2}(\mathbb{C})} \leq\left\|G\left(\sqrt{\widetilde{\square}_{\tau p}}\right)\right\|_{L^{2} \rightarrow L^{2}}\left\|K_{F}(z, \cdot)\right\|_{L^{2}(\mathbb{C})} .
$$

5.3. $L^{2}$-estimates of the resolvant and a decay estimate. As mentioned above, we need a decay result for the Fourier transform on the tail of a Gaussian in one dimension. Let $\ell \geq 1$ and $\varphi_{\ell} \in C^{\infty}(\mathbb{R})$ so that $\varphi_{\ell}$ is even, $\varphi_{\ell}(x)=1$ if $|x| \geq \ell$, and $\varphi(x)=0$ if $|x| \leq \frac{1}{2} \ell$. Also, let $0 \leq \varphi_{\ell} \leq 1$ and $\left|\varphi^{(n)}\right| \leq a_{n} \ell^{-n}$.

Proposition 5.7. For each $N \in \mathbb{N}$, there exists a constant $C_{N}$ so that if $\lambda \geq 0$ :

$$
\left|\mathcal{F}^{-1}\left[\varphi_{\ell} e^{-\frac{x^{2}}{4}}\right](\lambda)\right|=\left|\frac{1}{2 \pi} \int_{\mathbb{R}} \varphi_{\ell}(\xi) e^{-\frac{\xi^{2}}{4}} e^{i \lambda \xi} d \xi\right| \leq C_{N} e^{-\frac{\ell^{2}}{16}} \frac{1}{\left(\ell^{2}+\lambda^{2}\right)^{N / 2}} .
$$


Proof. The proof follows from an integration by parts. It is enough to estimate the integral on $[0, \infty)$. Integrating by parts $N$ times yields:

$$
\begin{aligned}
\int_{0}^{\infty} \varphi_{\ell}(\xi) e^{-\frac{\xi^{2}}{4}+i \lambda \xi} d \xi & =\int_{0}^{\infty}\left(\frac{1}{\xi / 2-i \lambda}\left(\cdots\left(\frac{1}{\xi / 2-i \lambda} \varphi_{\ell}(\xi)\right)^{\prime} \cdots\right)^{\prime}\right)^{\prime} e^{-\frac{\xi^{2}}{4}+i \lambda \xi} d \xi \\
& =\sum_{j=0}^{N} c_{j} \int_{0}^{\infty} \frac{1}{(\xi / 2-i \lambda)^{N+j}} \varphi_{\ell}^{(N-j)}(\xi) e^{-\frac{\xi^{2}}{4}+i \lambda \xi} d \xi .
\end{aligned}
$$

If $j=N$,

$$
\begin{aligned}
\left|\int_{\frac{\ell}{2}}^{\infty}(\xi / 2-i \lambda)^{-2 N} \varphi_{\ell}(\xi) e^{-\frac{\xi^{2}}{4}+i \lambda \xi} d \xi\right| & \leq e^{-\frac{\ell^{2}}{16}} \int_{\ell}^{\infty}\left|\frac{\xi}{2}-i \lambda\right|^{-2 N} d \xi \\
& \leq c_{N} e^{-\frac{\ell^{2}}{16}} \int_{\ell}^{\infty}(\xi+\lambda)^{-2 N} d \xi \\
& \leq c_{N} e^{-\frac{\ell^{2}}{16}}(\ell+\lambda)^{-2 N+1}=C_{N} e^{-\frac{\ell^{2}}{16}}\left(\ell^{2}+\lambda^{2}\right)^{-N+\frac{1}{2}}
\end{aligned}
$$

If $j<N$,

$$
\begin{aligned}
\left|\int_{0}^{\infty} \frac{1}{(\xi / 2-i \lambda)^{N+j}} \varphi_{\ell}^{(N-j)}(\xi) e^{-\frac{\xi^{2}}{4}+i \lambda \xi} d \xi\right| & \leq c_{N} e^{-\frac{\ell^{2}}{16}} \ell|\ell-i \lambda|^{-j-N} \ell^{-N+j} \\
& \leq c_{N} e^{-\frac{\ell^{2}}{16}}\left(\ell^{2}+\lambda^{2}\right)^{-N / 2-j / 2} \ell^{-N+j+1} .
\end{aligned}
$$

The $j=0$ case is the worst estimate from the previous two inequalities, and we see that

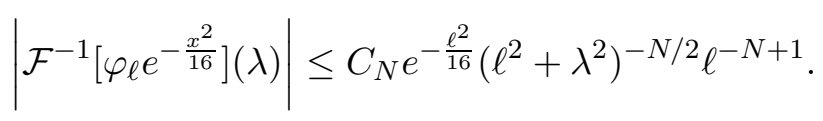

Next, we need $L^{2}$-bounds on the kernel of the resolvent $R\left(\lambda, \widetilde{\square}_{\tau p}\right)=\left(\lambda I+\widetilde{\square}_{\tau p}\right)^{-1}$. $\widetilde{\square}_{\tau p}$ has nonnegative eigenvalues, so $R\left(\lambda, \widetilde{\square}_{\tau p}\right)$ is a bounded, well-defined operator on $L^{2}(\mathbb{C})$ if $\lambda>0$. By the spectral theorem, if $\lambda>0$,

$$
R\left(\lambda, \widetilde{\square}_{\tau p}\right)[f]=\lim _{\epsilon \rightarrow 0} \int_{\epsilon}^{1 / \epsilon}\left(e^{-\lambda s} e^{-s \widetilde{\square}_{\tau p}}\right)[f] d s=\lim _{\epsilon \rightarrow 0} \int_{\epsilon}^{1 / \epsilon} e^{-\lambda s} H_{s}[f] d s .
$$

If $r_{\lambda}(z, w)$ is the integral kernel of $R\left(\lambda, \widetilde{\square}_{\tau p}\right)$, then it follows from (27) that

$$
r_{\lambda}(z, w)=\int_{0}^{\infty} e^{-\lambda s} \tilde{H}_{\tau p}(s, z, w) d s .
$$

We will need to compute $\int_{\mathbb{C}}\left|r_{\lambda}(z, w)\right|^{2} d A(w)$. A first step in that computation is

Proposition 5.8. Let $Y^{\alpha}$ be a product of operators $Y=X_{j}, j=1,2$ if acting in $z$ or $U_{j}, j=1,2$ if acting in $w$. Then there exists a constant $C_{|\alpha|}$ so that

$$
\left\|Y^{\alpha} \tilde{H}_{\tau p}(s, z, \cdot)\right\|_{L^{2}(\mathbb{C})} \leq C_{|\alpha|} \max \left\{s^{-\frac{1}{2}-\frac{1}{2}|\alpha|}, \mu_{p}(z, 1 / \tau)^{-1-|\alpha|}\right\} .
$$


Proof. Recall that $\tilde{H}_{\tau p}(s, z, w)=\tilde{G}_{\tau p}(s, z, w)+S_{\tau p}(z, w)$. By Corollary 2.5 and the change of variables that $x=c(z-w) / \mu(z, 1 / \tau)$, we have

$$
\begin{aligned}
\int_{\mathbb{C}}\left|Y^{\alpha} S_{\tau p}(z, w)\right|^{2} d A(w) & \lesssim \frac{1}{\mu(z, 1 / \tau)^{4+2|\alpha|}} \int_{\mathbb{C}} e^{-c \frac{|z-w|}{\mu(z, 1 / \tau)}} d A(w) \\
& =\frac{1}{\mu(z, 1 / \tau)^{2+2|\alpha|}} \int_{\mathbb{C}} e^{-|x|} d A(x) \sim \frac{1}{\mu(z, 1 / \tau)^{2+2|\alpha|}}
\end{aligned}
$$

Also, from Theorem 2.2 we see that one of the bounds on $\tilde{G}_{\tau p}(s, z, w)$ is (up to constants) the same bound for $S_{\tau p}(z, w)$ and hence already estimated. Also, integrating the Gaussian decay bound of $Y^{\alpha} \tilde{G}_{\tau p}(s, z, w)$, we have (with $\left.x=\sqrt{c}(z-w) / \sqrt{s}\right)$

$$
\int_{\mathbb{C}} \frac{1}{s^{2+|\alpha|}} e^{-c|z-w|^{2} / s} d A(w)=\frac{1}{s^{1+|\alpha|}} \int_{\mathbb{C}} e^{-|x|^{2}} d A(x) \sim \frac{1}{s^{1+|\alpha|}} .
$$

Taking the square roots of the estimates (29) and (30) finishes the proof.

Using Proposition 5.8, we can find an $L^{2}$ bound on $r_{\lambda}(z, w)$.

Proposition 5.9. There exists a constant $C$ so that

$$
\left\|r_{\lambda}(z, \cdot)\right\|_{L^{2}(\mathbb{C})} \leq C \max \left\{\frac{1}{\lambda \mu(z, 1 / \tau)}, \frac{1}{\lambda^{1 / 2}}\right\} .
$$

Proof. From (28) and Proposition 5.8, it follows from Minkowski's Inequality for Integrals that

$$
\begin{aligned}
\left(\int_{\mathbb{C}}\left|r_{\lambda}(z, w)\right|^{2} d A(w)\right)^{\frac{1}{2}} & =\left(\int_{\mathbb{C}}\left|\int_{0}^{\infty} e^{-\lambda s} \tilde{H}_{\tau p}(s, z, w) d s\right|^{2} d A(w)\right)^{\frac{1}{2}} \\
& \leq \int_{0}^{\infty} e^{-\lambda s}\left\|\tilde{H}_{\tau p}(s, z, \cdot)\right\| d s \leq \int_{0}^{\infty} e^{-\lambda s} \max \left\{s^{-1 / 2}, \mu_{p}(z, 1 / \tau)^{-1}\right\} d s .
\end{aligned}
$$

Certainly $\int_{0}^{\infty} e^{-\lambda s} \mu(z, 1 / \tau)^{-1} d s=\lambda^{-1} \mu(z, 1 / \tau)^{-1}$. Also,

$$
\int_{0}^{\infty} s^{-1 / 2} e^{-\lambda s} \leq \int_{0}^{1 / \lambda} s^{-1 / 2} d s+\int_{1 / \lambda}^{\infty} \lambda^{1 / 2} e^{-\lambda s} d s \sim \lambda^{-1 / 2} .
$$

5.4. Relationship between the heat and wave kernels and a decomposition of the heat kernel. The next part of our discussion explores the relationship between the heat kernel $\tilde{H}_{\tau p}(s, z, w)$ and the wave kernel $V_{\tau p}(s, z, w)$. If $f \in L^{1}(\mathbb{R})$,

$$
\hat{f}(\xi)=\int_{\mathbb{R}} e^{-i \xi x} f(x) d x .
$$

From this formula, it quickly follows that

$$
\frac{1}{2 \sqrt{\pi s}} \int_{0}^{\infty} e^{-r^{2} / 4 s} \cos r \xi d r=e^{-s \xi^{2}} .
$$

(31) is the basis for the relationship between the wave kernel and the heat kernel. Since $\widetilde{\square}_{\tau p}$ is self-adjoint and positive (the latter condition meaning $\left(\widetilde{\square}_{\tau p} f, f\right) \geq 0$ for all $\left.f \in \operatorname{Dom}\left(\widetilde{\square}_{\tau p}\right)\right)$, the 
spectral theorem implies that $\sqrt{\widetilde{\square}_{\tau p}}$ is a well-defined operator. Even further, the spectral theorem and (31) prove that

$$
\frac{1}{2 \sqrt{\pi s}} \int_{0}^{\infty}\left(\cos \left(r \sqrt{\widetilde{\square}_{\tau p}}\right) f_{1}, f_{2}\right) e^{-r^{2} / 4 s} d r=\left(e^{-s \widetilde{\square}_{\tau p}} f_{1}, f_{2}\right) .
$$

From the kernel side, equation (32) becomes

$$
\begin{array}{rl}
\frac{1}{2 \sqrt{\pi s}} \int_{0}^{\infty} \int_{\mathbb{C}} \int_{\mathbb{C}} V_{\tau p}(r, z, w) f_{1}(w) \overline{f_{2}(z)} e^{-r^{2} / 4 s} & d A(w) d A(z) d r \\
& =\int_{\mathbb{C}} \int_{\mathbb{C}} \tilde{H}_{\tau p}(s, z, w) f_{1}(w) \overline{f_{2}(z)} d A(w) d A(z) .
\end{array}
$$

Thus, from (33) and the support condition from Corollary 5.4, it follows that

\section{Proposition 5.10.}

$$
\tilde{H}_{\tau p}(s, z, w)=\frac{1}{2 \sqrt{\pi s}} \int_{0}^{\infty} V_{\tau p}(r, z, w) e^{-r^{2} / 4 s} d r=\frac{1}{2 \sqrt{\pi s}} \int_{|z-w|}^{\infty} V_{\tau p}(r, z, w) e^{-r^{2} / 4 s} d r .
$$

We are finally ready to decompose $e^{-s \widetilde{\square}_{\tau p}}$. Let $\ell>1$ (to be chosen later) and let

$$
\widehat{F_{\ell}^{\infty}}(\xi)=\sqrt{\pi} \varphi_{\ell}(\xi) e^{-\frac{\xi^{2}}{4}} \quad \text { and } \quad \widehat{F_{\ell}^{0}}(\xi)=\sqrt{\pi}\left(1-\varphi_{\ell}(\xi)\right) e^{-\frac{\xi^{2}}{4}} .
$$

Then $F_{\ell}^{0}$ and $F_{\ell}^{\infty}$ are bounded, even functions and

$$
e^{-s \widetilde{\square}_{\tau p}}=F_{\ell}^{0}\left(\sqrt{s \widetilde{\square}_{\tau p}}\right)+F_{\ell}^{\infty}\left(\sqrt{s \widetilde{\square}_{\tau p}}\right) .
$$

Next, $\operatorname{supp}\left(1-\varphi_{\ell}\right) \subset(-\ell, \ell)$, so by Proposition 5.5.

$$
\operatorname{supp} K_{F_{\ell}^{0}\left(\sqrt{s \widetilde{\square}_{\tau p}}\right)}(\cdot, \cdot) \subset\left\{(z, w):|z-w|<s^{1 / 2} \ell\right\} .
$$

Fix $z, w \in \mathbb{C}$ and $s>0$ so that $|z-w|>s^{\frac{1}{2}}$. Set $\ell=\ell(z, w)=|z-w| / s^{1 / 2}$. By (35),

$$
K_{F_{\ell}^{0}\left(\sqrt{\left.s \widetilde{\square}_{\tau p}\right)}\right.}(z, w)=0,
$$

SO

$$
\tilde{H}_{\tau p}(s, z, w)=K_{F_{\ell}^{\infty}\left(\sqrt{s \widetilde{\square}_{\tau p}}\right)}(z, w) .
$$

For simplicity, set $F(x)=F_{\ell}^{\infty}(x)$. Note that Proposition [5.7 applies to $F(x)$. For the next step in the argument, we would like to take $\sqrt{F}$, but that is unlikely to exist. While $F$ is $\mathbb{R}$-valued, there is no reason to believe that $F \geq 0$. Instead, we can write $F(\xi)=|F(\xi)| e^{i \theta(\xi)}$, and our replacement for the "square root" of $F$ is $f(\xi)=|F(\xi)|^{1 / 2} e^{i \theta(\xi) / 2}$ with our branch cut taken so that $0 \leq \theta<2 \pi$. In fact, $\theta(\xi)=0$ or $\pi$. From Proposition [5.7, we have (taking a square root of the estimate) that for all $N \geq 1$

$$
\sup _{\lambda \geq 0}\left|f(\lambda)\left(\ell^{2}+\lambda^{2}\right)^{N / 4}\right| \leq C_{N} e^{-c \ell^{2}}=C_{N} e^{-c \frac{|z-w|^{2}}{s}} .
$$

Next, we would like to use Proposition [5.6 and (37), our weighted $L^{\infty}$-estimate of $f$, to bound $\tilde{H}_{\tau p}(s, z, w)$. We have:

$$
\left|\tilde{H}_{\tau p}(s, z, w)\right|=\left|K_{F\left(\sqrt{s \widetilde{\square}_{\tau p}}\right)}(z, w)\right| \leq\left\|K_{f\left(\sqrt{s \widetilde{\square}_{\tau p}}\right)}(z, \cdot)\right\|_{L^{2}(\mathbb{C})}\left\|K_{f\left(\sqrt{s \widetilde{\square_{\tau p}}}\right)}(\cdot, w)\right\|_{L^{2}(\mathbb{C})}
$$


Evaluating each term separately, we have (with $\lambda=\sqrt{s \widetilde{\square}_{\tau p}}$ and $N=4$ ) by Proposition [5.6]

$$
\begin{aligned}
\left\|K_{f\left(\sqrt{\left.s \widetilde{\square}_{\tau p}\right)}\right.}(z, \cdot)\right\|_{L^{2}(\mathbb{C})} & =\left\|K_{f\left(\sqrt{s \widetilde{\square}_{\tau p}}\right)\left(\ell^{2} I+s \widetilde{\square}_{\tau p}\right)\left(\ell^{2} I+s \widetilde{\square}_{\tau p}\right)^{-1}}(z, \cdot)\right\|_{L^{2}(\mathbb{C})} \\
& \lesssim \sup _{\lambda>0}\left|f(\lambda)\left(\ell^{2}+\lambda^{2}\right)\right|\left\|K_{\left(\ell^{2}+s \widetilde{\square}_{\tau p}\right)^{-1}}(z, \cdot)\right\|_{L^{2}(\mathbb{C})} .
\end{aligned}
$$

Next, since $\ell=\frac{|z-w|}{s^{1 / 2}}>1$, from Proposition 5.9 we have

$$
\begin{aligned}
\left\|K_{\left(\ell^{2} I+s \widetilde{\square}_{\tau p}\right)^{-1}}(z, \cdot)\right\|_{L^{2}(\mathbb{C})} & =\left\|K_{\frac{1}{s}\left(\frac{\ell^{2}}{s} I+\widetilde{\square}_{\tau p}\right)^{-1}}(z, \cdot)\right\|_{L^{2}(\mathbb{C})}=\frac{1}{s}\left\|r_{\frac{\ell^{2}}{s}}(z, \cdot)\right\|_{L^{2}(\mathbb{C})} \\
& \lesssim \frac{1}{s} \max \left\{\frac{s}{\ell^{2} \mu(z, 1 / \tau)}, \frac{s^{1 / 2}}{\ell}\right\} \lesssim \max \left\{\frac{1}{\mu(z, 1 / \tau)}, \frac{1}{s^{1 / 2}}\right\} .
\end{aligned}
$$

Putting our estimates together finally allows us to prove

Theorem 5.11. There exists constants $c, C>0$ so that if $|z-w|>s^{1 / 2}$,

$$
\left|\tilde{H}_{\tau p}(s, z, w)\right| \leq C e^{-c \frac{|z-w|^{2}}{s}} \max \left\{\frac{1}{\mu_{p}(z, 1 / \tau) \mu_{p}(w, 1 / \tau)}, \frac{1}{s}\right\}
$$

For a discussion on a quantitative estimate of $c$, see [Sik04.

Proof. The result follows immediately from combining (38), (37), (39), and (40).

\section{Proof of Theorem 2.1}

We first prove off-diagonal estimates for $X_{z}^{\alpha} U_{w}^{\gamma} \tilde{H}_{\tau p}(s, z, w)$. We need the following result.

Lemma 6.1. Let $a, b>0,|z-w| \geq \mu_{p}(z, 1 / \tau)$, and $|z-w| \geq \mu_{p}(w, 1 / \tau)$. Then there exists $a$ constant $C_{a, b}$ so that

$$
\begin{aligned}
& e^{-c \frac{|z-w|^{2}}{s}} \max \left\{\frac{1}{s^{a}}, \frac{1}{\mu_{p}(z, 1 / \tau)^{2 a}}\right\} \cdot \max \left\{\frac{e^{-c \frac{s}{\mu_{p}(z, 1 / \tau)^{2}}}}{s^{b}}, \frac{e^{-c \frac{|z-w|}{\mu_{p}(z, 1 / \tau)}}}{\mu_{p}(z, 1 / \tau)^{2 b}}\right\} \\
& \leq C_{a, b} e^{-c \frac{|z-w|^{2}}{s}} \max \left\{\frac{e^{-c \frac{s}{\mu_{p}(z, 1 / \tau)^{2}}}}{s^{a+b}}, \frac{e^{-c \frac{|z-w|}{\mu_{p}(z, 1 / \tau)}}}{\mu_{p}(z, 1 / \tau)^{2(a+b)}}\right\} .
\end{aligned}
$$

Proof. If $s^{-1} \geq \mu_{p}(z, 1 / \tau)^{-2}$ and $\frac{e^{-c \frac{s}{\mu_{p}(z, 1 / \tau)^{2}}}}{s^{b}} \geq \frac{e^{-c \frac{|z-w|}{\mu_{p}(z, 1 / \tau)}}}{\mu_{p}(z, 1 / \tau)^{2 b}}$ or $s^{-1} \leq \mu_{p}(z, 1 / \tau)^{-2}$ and $\frac{e^{-c \frac{s}{\mu_{p}(z, 1 / \tau)^{2}}}}{s^{b}} \leq$ $\frac{e^{-c \frac{|z-w|}{\mu_{p}(z, 1 / \tau)}}}{\mu_{p}(z, 1 / \tau)^{2 b}}$, then there is nothing to prove. Thus we have two cases to consider. Assume that $s^{-1} \geq \mu_{p}(z, 1 / \tau)^{-2}$ and $\frac{e^{-c \frac{s}{\mu_{p}(z, 1 / \tau)^{2}}}}{s^{b}} \leq \frac{e^{-c \frac{|z-w|}{\mu_{p}(z, 1 \mid \tau)}}}{\mu_{p}(z, 1 / \tau)^{2 b}}$. Note that with a decrease in $c, e^{-c \frac{|z-w|^{2}}{s}} \leq$ $\frac{s^{a}}{|z-w|^{2 a}}|z-w| e^{-c \frac{|z-w|^{2}}{s}}$. Since $|z-w| \geq \mu_{p}(z, 1 / \tau)$,

$$
e^{-c \frac{|z-w|^{2}}{s}} \frac{1}{s^{a}} \frac{e^{-c \frac{|z-w|}{\mu_{p}(z, 1 / \tau)}}}{\mu_{p}(z, 1 / \tau)^{2 b}} \leq \frac{s^{a}}{|z-w|^{2 a}} e^{-c \frac{|z-w|^{2}}{2 s}} \frac{1}{s^{a}} \frac{e^{-c \frac{|z-w|}{\mu_{p}(z, 1 / \tau)}}}{\mu_{p}(z, 1 / \tau)^{2 b}} .
$$


Now assume that $s^{-1} \leq \mu_{p}(z, 1 / \tau)^{-2}$ and $\frac{e^{-c \frac{s}{\mu_{p}(z, 1 / \tau)^{2}}}}{s^{b}} \geq \frac{e^{-c \frac{|z-w|}{\mu_{p}(z, 1 / \tau)}}}{\mu_{p}(z, 1 / \tau)^{2 b}}$. The argument is similar to the first case, except we use the fact that $e^{-c \frac{s}{\mu_{p}(z, 1 / \tau)^{2}}} \lesssim \frac{\mu_{p}(z, 1 / \tau)^{2 a}}{s^{a}} e^{-c \frac{s}{\mu_{p}(z, 1 / \tau)^{2}}}$ with a decrease in the constant $c$.

The bound on $X_{z}^{\alpha} U_{w}^{\gamma} \tilde{H}_{\tau p}(s, z, w)$ is proven in two stages. We first prove:

Lemma 6.2. Let $z, w \in \mathbb{C}$ so that $|z-w| \geq 2 \mu_{p}(z, 1 / \tau)$ and $|z-w| \geq 2 \mu_{p}(w, 1 / \tau)$. Let $X_{z}^{\alpha}$ be a product of $|\alpha|$ operators of the form $X=X_{j}, j=1,2$ acting in the $z$ variable. Then there exists a constant $C=C_{|\alpha|}$ so that

$$
\left|X_{z}^{\alpha} \tilde{H}_{\tau p}(s, z, w)\right| \leq C e^{-c \frac{|z-w|^{2}}{s}} \max \left\{\frac{e^{-c \frac{s}{\mu_{p}(z, 1 / \tau)^{2}}}}{s^{1+\frac{1}{2}|\alpha|}}, \frac{e^{-c \frac{|z-w|}{\mu_{p}(z, 1 / \tau)^{2}}}}{\mu_{p}(z, 1 / \tau)^{2+|\alpha|}}\right\}
$$

Proof. The on-diagonal result for $|\alpha|=0$ follows quickly from Theorem 2.2. Corollary [2.5, and Lemma 6.1. The off-diagonal result follows immediately from Theorem[5.11] Theorem 2.2, Corollary 2.5] and Lemma 6.1]

We turn our attention to the case $|\alpha| \geq 1$. Let $\delta=\min \left\{s^{1 / 2}, \mu_{p}(z, 1 / \tau)\right\}$. Let $\varphi_{z} \in \mathcal{C}_{c}^{\infty}(\mathbb{C})$ so that $\varphi_{z} \equiv 1$ on $D(z, \delta), \varphi \equiv 0$ off of $D\left(z, \frac{3}{2} \delta\right)$, and $\left|D^{n} \varphi_{z}\right| \leq c_{n} \delta^{-n}$. By Theorem 3.1.

$$
\begin{aligned}
& \left|X_{z}^{\alpha} \tilde{H}_{\tau p}(s, z, w)\right| \lesssim \frac{1}{\delta} \sum_{|I| \leq 2} \delta^{|I|}\left\|X_{z}^{I}\left[X_{z}^{\alpha} \tilde{H}_{\tau p}(s, \cdot, w) \varphi_{z}\right]\right\|_{L^{2}(\mathbb{C})} \\
& \quad \leq \frac{1}{\delta} \sum_{|I| \leq 2} \sum_{|J| \leq|I|} \delta^{|I|}\left\|X_{z}^{J} X_{z}^{\alpha} \tilde{H}_{\tau p}(s, \cdot, w) D^{|I|-|J|} \varphi_{z}\right\|_{L^{2}(\mathbb{C})} \\
& \quad=\frac{1}{\delta} \sum_{|I| \leq 2} \sum_{|J| \leq|I|} \delta^{|I|}\left|\left(X_{z}^{\alpha} X_{z}^{J}\left(X_{z}^{J} X_{z}^{\alpha} \tilde{H}_{\tau p}(s, \cdot, w) D^{|I|-|J|} \varphi_{z} \overline{D^{|I|-|J|} \varphi_{z}}\right), \tilde{H}_{\tau p}(s, \cdot, w)\right)\right|^{\frac{1}{2}} \\
& \quad \leq \frac{1}{\delta} \sum_{\substack{|I| \leq 2 \\
|J| \leq|I|}} \delta^{|I|}\left\|X_{z}^{J_{1}} X_{z}^{J} X_{z}^{\alpha} \tilde{H}_{\tau p}(s, \cdot, w) D^{|I|-|J|+\left|J_{2}\right|} \varphi_{z} D^{|I|-|J|+\left|J_{3}\right|} \varphi_{z}\right\|_{L^{2}(\mathbb{C})}^{\frac{1}{2}}\left\|\tilde{H}_{\tau p}(s, \cdot, w)\right\|_{L^{2}\left(\operatorname{supp} \varphi_{z}\right)}^{\frac{1}{\mid}} \| X_{1}|+| J_{2}|+| J_{3}|=| J|+| \alpha \mid \\
& \quad \leq \frac{1}{\delta} \sum_{\substack{|I| \leq 2 \\
|| I|\leq| I \mid}} \delta^{|I|} \frac{1}{\delta^{\frac{1}{2}\left(|I|-|J|+\left|J_{2}\right|+|I|-|J|+\left|J_{3}\right|\right)}}\left\|X_{z}^{J} X_{z}^{\alpha} \tilde{H}_{\tau p}(s, \cdot, w)\right\|_{L^{2}(\mathbb{C})}^{\frac{1}{2}}\left\|\tilde{H}_{\tau p}(s, \cdot, w)\right\|_{L^{2}\left(\operatorname{supp} \varphi_{z}\right) \cdot}^{\frac{1}{2}} \\
& \left|J_{1}\right|+\left|J_{2}\right|+\left|J_{3}\right|=|J|+|\alpha|
\end{aligned}
$$

The choice of $\delta$ is small enough so that

$$
\left\|\tilde{H}_{\tau p}(s, \cdot, w)\right\|_{L^{2}\left(\operatorname{supp} \varphi_{z}\right)} \lesssim \delta \sup _{\zeta \in D\left(z, \frac{3}{2} \delta\right)}\left|\tilde{H}_{\tau p}(s, \zeta, w)\right| \sim \delta\left|\tilde{H}_{\tau p}(s, z, w)\right|
$$

with a decrease in the constants $c_{j}$. The estimate from the other terms follows from Proposition 5.8. and we see

$$
\begin{aligned}
\left\|X_{z}^{J_{1}} X_{z}^{J} X_{z}^{\alpha} \tilde{H}_{\tau p}(s, \cdot, w)\right\|_{L^{2}(\mathbb{C})} & \leq \max \left\{\frac{1}{s^{\frac{1}{2}\left(1+|\alpha|+|J|+\left|J_{1}\right|\right)}}, \frac{1}{\mu_{p}(z, 1 / \tau)^{1+|\alpha|+|J|+\left|J_{1}\right|}}\right\} \\
& =\delta^{-1-|\alpha|-|J|-\left|J_{1}\right|} .
\end{aligned}
$$


Combining our previous three estimates and using the fact we have already proved the result for $\alpha=0$, we have

$$
\begin{aligned}
& \left|X_{z}^{\alpha} \tilde{H}_{\tau p}(s, z, w)\right| \\
& \leq \frac{1}{\delta} \sum_{\substack{|I| \leq 2 \\
|J| \leq|I|}} \delta^{|I|} \delta^{-|I|+|J|-\frac{1}{2}\left(\left|J_{2}\right|+\left|J_{3}\right|+1+|\alpha|+|J|+\left|J_{1}\right|\right)} \delta^{\frac{1}{2}} e^{-c \frac{|z-w|^{2}}{s}} \max \left\{\frac{e^{-c \frac{s}{\mu_{p}(z, 1 / \tau)^{2}}}}{s^{\frac{1}{2}}}, \frac{e^{-c \frac{|z-w|}{\mu_{p}(z, 1 / \tau)^{2}}}}{\mu_{p}(z, 1 / \tau)}\right\} \\
& \lesssim \frac{1}{J_{1}|+| J_{2}|+| J_{3}|=| J|+| \alpha \mid} e^{-c \frac{|z-w|^{2}}{s}} \max \left\{\frac{e^{-c \frac{s}{\mu_{p}(z, 1 / \tau)^{2}}}}{s^{\frac{1}{2}}}, \frac{e^{-c \frac{|z-w|}{\mu_{p}(z, 1 / \tau)^{2}}}}{\mu_{p}(z, 1 / \tau)}\right\} .
\end{aligned}
$$

Since $\frac{1}{\delta}=\max \left\{\frac{1}{s^{1 / 2}}, \frac{1}{\mu_{p}(z, 1 / \tau)}\right\}$, the estimate simplifies to

$$
\left|X_{z}^{\alpha} \tilde{H}_{\tau p}(s, z, w)\right| \lesssim \max \left\{\frac{1}{s^{1 / 2+\frac{1}{2}|\alpha|}}, \frac{1}{\mu_{p}(z, 1 / \tau)^{1+|\alpha|}}\right\} e^{-c \frac{|z-w|^{2}}{s}} \max \left\{\frac{e^{-c \frac{s}{\mu_{p}(z, 1 / \tau)^{2}}}}{s^{\frac{1}{2}}}, \frac{e^{-c \frac{|z-w|}{\mu_{p}(z, 1 / \tau)^{2}}}}{\mu_{p}(z, 1 / \tau)}\right\} .
$$

From Lemma 6.1 it follows that

$$
\left|X_{z}^{\alpha} \tilde{H}_{\tau p}(s, z, w)\right| \lesssim e^{-c \frac{|z-w|^{2}}{s}} \max \left\{\frac{e^{-c \frac{s}{\mu_{p}(z, 1 / \tau)^{2}}}}{s^{1+\frac{1}{2}|\alpha|}}, \frac{e^{-c \frac{|z-w|}{\mu_{p}(z, 1 / \tau)^{2}}}}{\mu_{p}(z, 1 / \tau)^{2+|\alpha|}}\right\}
$$

Proof (Theorem [2.1) $)$. Since $\tilde{H}_{\tau p}(s, z, w)=\tilde{G}_{\tau p}(s, z, w)+S_{\tau p}(z, w)$, the on-diagonal estimates $(\mid z-$ $\left.w \mid \leq 2 \mu_{p}(z, 1 / \tau)\right)$ follow from the bounds of the Szegö kernel and Theorem 2.2

By Theorem 4.3, the only case left to consider is the bound for $\left|Y^{\alpha} \tilde{H}_{\tau p}(s, z, w)\right|$. We can write $Y^{\alpha}=X_{w}^{\beta} U_{w}^{\gamma}$ for some multi-indices $\beta$ and $\gamma$. Also, from Theorem 6.2 and the fact that $\tilde{H}_{\tau p}(s, z, w)=\overline{\tilde{H}}_{\tau p}(s, w, z)$, it follows that we have the desired estimate for $\left|U_{w}^{\gamma} \tilde{H}_{\tau p}(s, z, w)\right|=$ $\left|\overline{X_{w}^{\gamma} \tilde{H}_{\tau p}(s, w, z)}\right|$. Repeating the argument of Lemma 6.2 with $U_{w}^{\gamma} \tilde{H}_{\tau p}(s, z, w)$ taking the place of $\tilde{H}_{\tau p}(s, z, w)$ finishes the proof.

\section{REFERENCES}

[Ada78] Robert Adams. Sobolev Spaces, volume 65 of Pure and applied mathematics. Academic Press [A subsidiary of Harcourt Brace Jovanovich, Publishers], New York-London, 1978.

[Ber94] B. Berndtsson. Some recent results on estimates for the $\bar{\partial}$-equation. In Contributions to complex analysis and analytic geometry, 27-42 (H. Skoda and J. M. Trépreau, eds.), Aspects Math., vol. E26. Vieweg, Braunschweig, 1994.

[Ber96] B. Berndtsson. $\bar{\partial}$ and Schrödinger operators. Math. Z., 221:401-413, 1996.

[CF05] M. Christ and S. Fu. Compactness in the $\bar{\partial}$-Neumann problem, magnetic Schrödinger operators, and the Aharonov-Bohm effect. Adv. Math., 197:1-40, 2005.

[Chr91] M. Christ. On the $\bar{\partial}$ equation in weighted $L^{2}$ norms in $\mathbb{C}^{1}$. J. Geom. Anal., 1(3):193-230, 1991.

[FS91] J.E. Fornæss and N. Sibony. On $L^{p}$ estimates for $\bar{\partial}$. In Several complex variables and complex geometry, Part 3 (Santa Cruz, CA, 1989), Proc. Sympos. Pure Math., 52, Part 3, pages 129-163, Providence, R.I., 1991. American Mathematical Society.

[FS02] S. Fu and E. Straube. Semi-classical analysis of Schrödinger operators and compactness in the $\bar{\partial}$-Neumann problem. J. Math. Anal. Appl., 271:267-282, 2002. 
[FS03] S. Fu and E. Straube. Correction to: "Semi-classical analysis of Schrödinger operators and compactness in the $\bar{\partial}$-Neumann problem". J. Math. Anal. Appl., 280:195-196, 2003.

[Kur00] K. Kurata. An estimate on the heat kernel of magnetic Schrödinger operators and uniformly elliptic operators with non-negative potentials. J. London Math. Soc., 62(3):885-903, 2000.

[Lig89] E. Ligocka. On the Forelli-Rudin construction and weighted Bergman projections. Studia Math., 94:257$272,1989$.

[NRSW89] A. Nagel, J.-P. Rosay, E.M. Stein, and S. Wainger. Estimates for the Bergman and Szegö kernels in $\mathbb{C}^{2}$. Ann. of Math., 129:113-149, 1989.

[NS] A. Nagel and E.M. Stein. The $\bar{\partial}_{b}$-complex on decoupled domains in $\mathbb{C}^{n}, n \geq 3$. To appear, Ann. of Math. (2).

[NS01] A. Nagel and E.M. Stein. The $\square_{b}$-heat equation on pseudoconvex manifolds of finite type in $\mathbb{C}^{2}$. Math. Z., 238:37-88, 2001.

[NS04] A. Nagel and E.M. Stein. On the product theory of singular integrals. Rev. Mat. Iberoamericana, 20:531561,2004 .

[NSW85] A. Nagel, E.M. Stein, and S. Wainger. Balls and metrics defined by vector fields I: Basic properties. Acta Math., 155:103-147, 1985.

[Rai05a] Andrew Raich. Heat Equations on $\mathbb{R} \times \mathbb{C}$. PhD thesis, University of Wisconsin-Madison, Department of Mathematics, 2005.

[Rai05b] Andrew Raich. Heat Equations in $\mathbb{R} \times \mathbb{C}$. submitted 2005. [arXiv:math.CV/0508571].

[Rai06] Andrew Raich. One-Parameter Families of Operators in C. J. Geom. Anal., 16(2):353-374, 2006.

[RS80] M. Reed and B. Simon. Methods of modern mathematical physics. I. Functional Analysis. Academic Press [Harcourt Brace Jovanovich, Publishers], New York-London, revised and Enlarged edition edition, 1980.

[Rud91] Walter Rudin. Functional Analysis. International Series in Pure and Applied Mathematics. McGraw-Hill, Inc., New York, Second edition, 1991.

[She96] Z. Shen. Estimates in $L^{p}$ for magnetic Schrödinger operators. Indiana Univ. Math. J., 45(3):817-841, 1996.

[She99] Z. Shen. On fundamental solutions of generalized Schrödinger operators. J. Funct. Anal., 167(2):521-564, 1999.

[Sik04] A. Sikora. Riesz transform, Gaussian bounds and the method of the wave equation. Math. Z., 247:643-662, 2004 .

Department of Mathematics, Texas A\&M University, Mailstop 3368, College Station, TX 778433368 